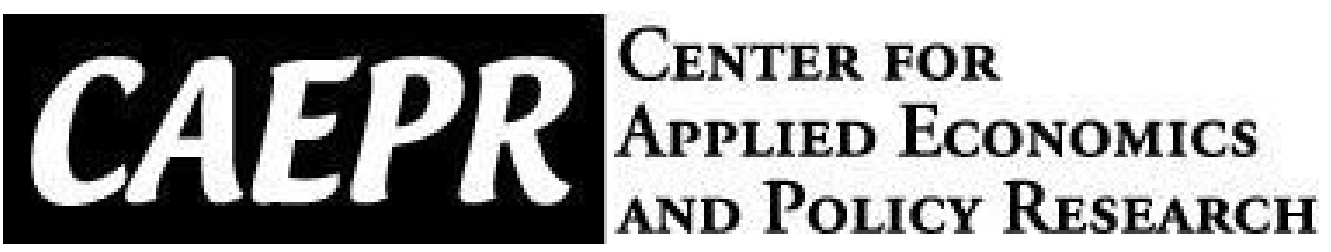

\author{
CAEPR \\ Working Paper \\ \#2018-011
}

\title{
Origins of Monetary Policy Shifts: A New Approach to Regime Switching in DSGE Models
}

\author{
Yoosoon Chang \\ Indiana University \\ Fei Tan, Dept. of \\ Economics \\ Chaifetz School of \\ Business, Saint Louis \\ University and Center for \\ Economic Behavior and \\ Decision-Making, \\ Zhejiang University of \\ Finance and Economics \\ Junior Maih \\ Norges Bank
}

February 17, 2021

This paper can be downloaded without charge from the Social Science Research

Network electronic library at https://papers.ssrn.com/sol3/papers.cfm?abstract_id=3290704

The Center for Applied Economics and Policy Research resides in the Department of Economics at Indiana University Bloomington. CAEPR can be found on the Internet at: http://www.indiana.edu/ -caepr. CAEPR can be reached via email at caepr@indiana.edu or via phone at 812-855-4050.

(C)2018 by Yoosoon Chang, Junior Maih and Fei Tan. All rights reserved. Short sections of text, not to exceed two paragraphs, may be quoted without explicit

permission provided that full credit, including (C) notice, is given to the source. 


\title{
Origins of Monetary Policy Shifts: A New Approach to Regime Switching in DSGE Models ${ }^{\dagger}$
}

\author{
Yoosoon Chang, Junior Maih, and Fei Tan*
}

[This Version: February 17, 2021]

\begin{abstract}
We examine monetary policy shifts by taking a new approach to regime switching in a small scale monetary DSGE model with threshold-type switching in the monetary policy rule. The policy response to inflation is allowed to switch endogenously between two regimes, hawkish and dovish, depending on whether a latent regime factor crosses a threshold level. Endogeneity stems from the historical impacts of structural shocks driving the economy on the regime factor. We quantify the endogenous feedback from each structural shock to the regime factor to understand the sources of the observed policy shifts. This new channel sheds new light on the interaction between policy changes and measured economic behavior. We develop a computationally efficient filtering algorithm for state-space models with time-varying transition probabilities that handles classical regression models as a special case. We apply this filter to estimate our DSGE model using the U.S. data and find strong evidence of endogeneity in the monetary policy shifts.
\end{abstract}

Keywords: Monetary policy, DSGE model, regime switching, latent autoregressive regime factor, endogenous feedback, expectation formation effects

JEL Classification: E52, C13, C32

\footnotetext{
${ }^{\dagger}$ This Working Paper should not be reported as representing the views of Norges Bank. The views expressed are those of the authors and do not necessarily reflect those of Norges Bank. An earlier draft of the paper was circulated as Chang et al. (2018a) under the title "State Space Models with Endogenous Regime Switching".

* Chang: Department of Economics, Indiana University; Maih: Norges Bank and BI Norwegian Business School; Tan: Department of Economics, Chaifetz School of Business, Saint Louis University and Center for Economic Behavior and Decision-Making, Zhejiang University of Finance and Economics.
} 


\section{INTRODUCTION}

In time series analysis, there is a long tradition in modeling structural change as the outcome of a regime switching process [Hamilton $(1988,1989)]$. By introducing an unobserved discrete-state Markov chain governing the regime in place, this class of models affords a tractable framework for the empirical analysis of time-varying dynamics that is endemic to many economic and financial phenomena. ${ }^{1}$

Despite the popularity of the Markov switching approach, its dynamics are ultimately governed by a regime switching process that is exogenous. This is especially unsatisfactory if we seek to truly understand the nature of policy making and its impact on economic phenomena. As argued in Chang et al. (2017), the presence of endogeneity in regime switching is indeed ubiquitous ${ }^{2}$ and, if ignored, may yield substantial biases and significantly deteriorate the precision in model parameter estimates. It follows that a more desirable approach to modeling occasional but recurrent regime shifts would admit some form of endogenous feedback from the behavior of underlying economic fundamentals to the regime generating process [Diebold et al. (1994), Chib and Dueker (2004), Kim (2004, 2009), Kim et al. (2008), Bazzi et al. (2014), Kang (2014), Kalliovirta et al. (2015), Kim and Kim (2018), among others].

The purpose of this paper is to introduce a threshold-type endogenous regime switching framework into dynamic linear models that can be represented in state space forms. This class of models is broad, including classical regression models and the popular dynamic stochastic general equilibrium (DSGE) models as special cases, and thus allows for a greater scope for understanding the complex interaction between regime switching and measured economic behavior.

Following Chang et al. (2017), an essential feature of our model is that the data generating process alternates between two regimes, depending on whether an autoregressive latent factor crosses some threshold level. ${ }^{3}$ In our approach, two sources of random innovations jointly drive the latent factor and hence the regime change: (i) the internal innovations from the transition equation that represent the fundamental shocks inside the model; (ii) an external innovation that captures all other shocks

\footnotetext{
${ }^{1}$ Among recent developments of this approach, Kim (1994) made an important extension to the state space representation of dynamic linear models amenable to classical inference, whereas Chib (1996) presented a full Bayesian analysis for finite mixture models based on Gibbs sampling. An introductory exposition and overview of the related literature can be found in the monograph by Kim and Nelson (1999).

${ }^{2}$ This endogeneity can be illustrated for instance by the fact that central banks switch to unconventional policies when the policy rate becomes constrained by the zero-lower bound. Another example relates to to mortgagors being subject to stringent borrowing conditions either when credit growth has been excessive or when there is a downturn in the economy. Binning and Maih (2017) present a general framework for modeling occasionally-binding constraints using regime switching. More recently, Benigno et al. (2020) apply similar techniques to document endogenous switches into and out of financial crises in Mexico.

${ }^{3}$ Our approach and its supporting software is not bound by the assumption of only two regimes. By introducing either multiple regime factors or threshold levels, the model can switch among more than two regimes.
} 
left outside the model. The relative importance of the former source determines the degree of endogeneity in regime changes. The autoregressive nature of the latent factor, on the other hand, makes such endogenous effects long-lasting - a current shock to the transition equation will impact at a decaying rate on all future latent factors. Most importantly, regime switching of this type renders the transition probabilities as time-varying in that they are all functions of the model's fundamentals. In the special case where regime shifts are purely driven by the external innovation, our model becomes observationally equivalent to one with conventional Markov switching.

The main contributions of this paper are twofold, one methodological and the other substantive. This paper develops an approximate endogenous-switching filter based on the algorithms of Kim (1994) and Chang et al. (2017) to estimate the overall nonlinear state space model. Calculations are simplified by an appropriate augmentation of the transition equation and exploiting the conditionally linear and Gaussian structure. Unlike simulation-based filters, this avoids sequential Monte Carlo integration and as such makes our filter computationally efficient. As a useful by-product of running the filter, the estimated autoregressive latent factor can be readily constructed from the filter outputs. ${ }^{4}$

The substantive contribution of this paper is to provide a framework within which we study the origins of monetary policy shifts. Ever since the seminal work of Clarida et al. (2000), modeling the time-varying behavior of monetary policy has remained an active research agenda for macroeconomists. Figure 1 displays prima facie evidence of such time variation. Panel A makes clear that the Taylor rule for setting the federal funds rate provides by and large an accurate account of the postwar U.S. monetary policy. Nevertheless, there exist several persistent and sizable discrepancies as shown in Panel B-monetary interventions (i.e., surprise changes in the policy rate) reflecting policy considerations beyond the Taylor rule mandates. Most evident is the sustained and dramatic loosening of policy under Federal Reserve chairmen Arthur Burns and G. William Miller in the early and late 1970s, followed by several severe tightening of policy to fight the Great Inflation under Paul Volcker in the early 1980s. Economic agents who observe this drastic policy change would shift their beliefs about monetary policy to a more aggressive regime for controlling the inflation.

While regime switching has emerged as a promising approach to modeling the time variation in monetary policy, scant attention in the literature has been paid to the macroeconomic origins that give rise to monetary policy shifts over time. Our paper takes a first step toward filling in this important gap - the aim here is not to identify policy switches that standard approaches would miss but rather to shed light into the reasons why the switching occurs. For pedagogical purposes, we first employ a Fisherian model of inflation determination to endogenize regime change

\footnotetext{
${ }^{4}$ The filter is developed for the econometrician to evaluate the model's likelihood function. The latent factor itself, however, is not (explicitly) filtered by the economic agents. Having observed the history of shocks, and knowing the structure of the latent factor, the economic agents proceed to compute the probability that a regime switch will occur, which helps them make the economic decisions.
} 
A. Federal Funds Rate

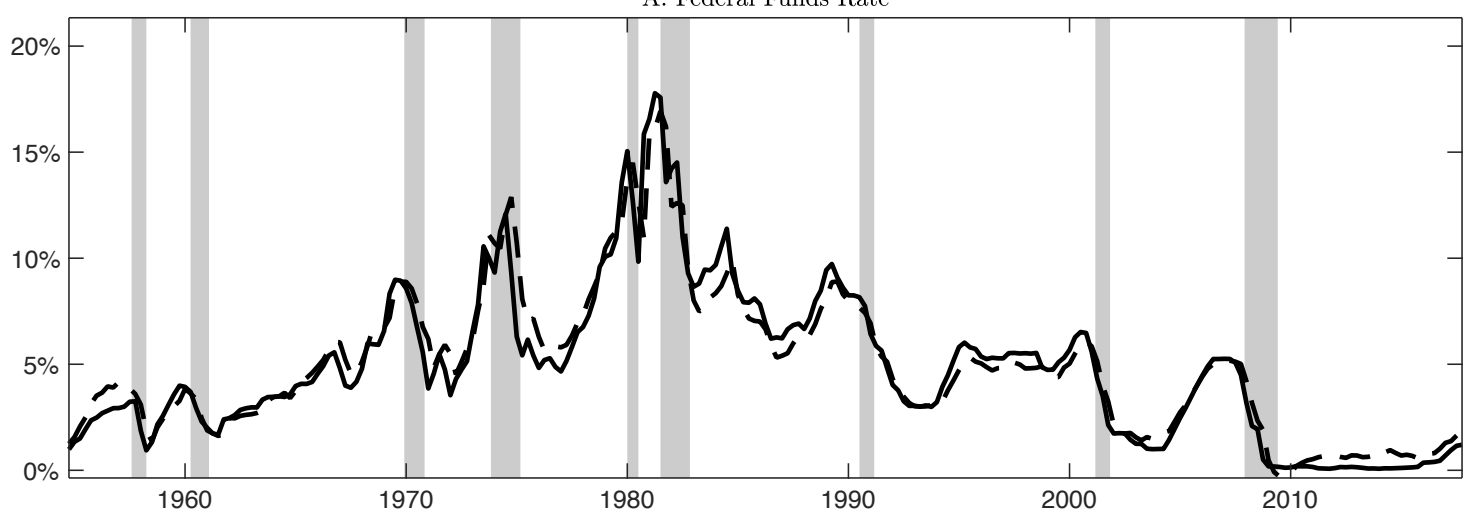

B. Monetary Policy Intervention

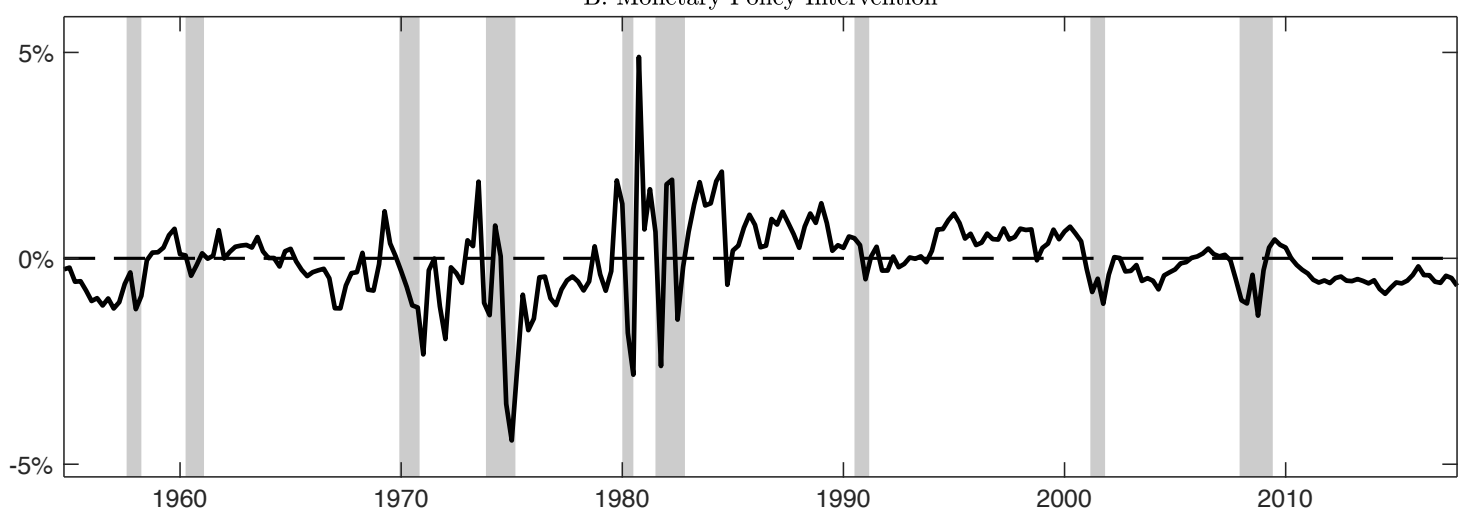

Figure 1: Federal funds rate and monetary policy intervention. Notes: Panel A plots the effective federal funds rate (solid line) and the rate implied by an inertial version of the Taylor (1993) rule (dashed line), $i_{t}=\rho i_{t-1}+(1-\rho)\left[4+1.5\left(\pi_{t}-2\right)+0.5 y_{t}\right]$, where $i$ denotes the federal funds rate, $\pi$ the annual inflation rate, $y$ the percentage deviation of real output from its potential, and $\rho=0.75$. Panel B depicts their differential. Shaded bars indicate recessions as designated by the National Bureau of Economic Research.

in monetary policy. The specification is simple enough to admit an analytical solution that makes transparent the mechanism at work, but also rich enough to highlight the general features of a rational expectations model with threshold-type endogenous switching. We then extend the simple model to a prototypical new Keynesian DSGE model whose state space form can be analyzed with our filter, and find that aggregate demand shocks play an important role in triggering the historical regime changes in the postwar U.S. monetary policy. To the best of our knowledge, modeling and quantifying such endogenous feedback channel are novel in the literature.

The rest of the paper is organized as follows. Section 2 describes the state space model and filtering algorithm. Section 3 adopts a simple analytical model to illustrate how to endogenize regime change in monetary policy using our endogenous-switching framework. Section 4 extends the simple model to an empirical DSGE model and derives its state space form that can be analyzed with our filtering algorithm. Section 5 concludes. 


\section{Model and Algorithm}

This section introduces the threshold-type endogenous switching framework, which extends that of Chang et al. (2017) and nests the conventional Markov switching as a special case, into the state space form of a general dynamic linear model. Like any regime switching model, the associated likelihood function depends on all possible histories of the entire regime path. This history-dependent nature creates a tight upper bound on the sample size that any exact recursive filter can comb through within a reasonable amount of time. ${ }^{5}$ Any pragmatic solution will inevitably require some approximations. Building on the 'collapsing' method of Kim (1994) to truncate the full historydependence, we develop an endogenous-switching version of the Kalman filter to approximate the likelihood function and estimate the unknown parameters as well as the state variables, including the autoregressive latent factor.

Throughout this section, we employ the following notation. Let $\mathbb{N}(\mu, \Sigma)$ denote the normal distribution with mean vector $\mu$ and covariance matrix $\Sigma, p_{\mathbb{N}}(\cdot \mid \mu, \Sigma)$ its probability density function, and $\Phi(\cdot)$ the cumulative distribution function of $\mathbb{N}(0,1)$. In particular, $\mathbb{N}\left(0_{n \times 1}, \mathrm{I}_{n}\right)$ denotes the $n$ dimensional standard normal distribution. Moreover, $p(\cdot \mid \cdot)$ and $\mathbb{P}(\cdot \mid \cdot)$ denote the conditional density and probability functions, respectively. Lastly, $Y_{1: T}$ is a matrix that collects the sample for periods $t=1, \ldots, T$ with row observations $y_{t}^{\prime}$.

2.1 State Space Model Let $y_{t}$ be an $l \times 1$ vector of observable variables, $x_{t}$ an $m \times 1$ vector of latent state variables, and $z_{t}$ a $k \times 1$ vector of predetermined explanatory variables. Consider the following regime-dependent linear state space model

$$
\begin{aligned}
& y_{t}=D_{s_{t}}+Z_{s_{t}} x_{t}+F_{s_{t}} z_{t}+\Omega_{s t}^{1 / 2} u_{t}, \quad u_{t} \sim \mathbb{N}\left(0_{l \times 1}, \mathrm{I}_{l}\right) \\
& x_{t}=C_{s_{t}}+G_{s_{t}} x_{t-1}+E_{s_{t}} z_{t}+M_{s_{t}} \Sigma_{s_{t}}^{1 / 2} \epsilon_{t}, \quad \epsilon_{t} \sim \mathbb{N}\left(0_{n \times 1}, \mathrm{I}_{n}\right)
\end{aligned}
$$

where the measurement equation (2.1) links the observable variables to the state variables subject to an $l \times 1$ vector of measurement errors $\Omega_{s_{t}}^{1 / 2} u_{t}$, the transition equation (2.2) describes the evolution of the state variables driven by an $n \times 1$ vector of exogenous innovations $\Sigma_{s_{t}}^{1 / 2} \epsilon_{t}$, and $\left(u_{t}, \epsilon_{t}\right)$ are mutually and serially uncorrelated at all leads and lags. The coefficient matrices $(D ., Z$., F., C., G., E., M.) and the covariance matrices $\left(\Omega ., \Sigma\right.$.) are both allowed to depend on an index variable $s_{t}=\mathbb{1}\left\{w_{t} \geqslant \tau\right\}$ driven by a stationary autoregressive latent factor

$$
w_{t}=\alpha w_{t-1}+v_{t}, \quad v_{t} \sim \mathbb{N}(0,1)
$$

\footnotetext{
${ }^{5}$ As noted by Kim (1994), even with two regimes, there would be over 1000 cases to consider by period $t=10$.
} 
where $-1<\alpha<1$ controls the persistency of $w_{t} \cdot{ }^{6}$ As a result, the model is switching between regime- 0 and regime- 1 , depending upon whether $w_{t}$ takes a value below or above the threshold level $\tau$. In what follows, we call $w_{t}$ the regime factor.

We allow all current standardized transition innovations $\epsilon_{t}$ to jointly influence the next period regime through their correlations with the innovation $v_{t+1}$ to $w_{t+1}$. Specifically,

$$
\left(\begin{array}{c}
\epsilon_{t} \\
v_{t+1}
\end{array}\right) \sim \mathbb{N}\left(\left(\begin{array}{c}
0_{n \times 1} \\
0
\end{array}\right),\left(\begin{array}{cc}
\mathrm{I}_{n} & \rho \\
\rho^{\prime} & 1
\end{array}\right)\right), \quad \rho^{\prime} \rho<1
$$

where $\rho=\left[\rho_{1}, \ldots, \rho_{n}\right]^{\prime}=\operatorname{corr}\left(\epsilon_{t}, v_{t+1}\right)$ is a vector of correlation parameters that determines the degree of endogeneity in regime changes - as $\rho$ approaches to one in modulus, today's transition innovations impinge more forcefully on tomorrow's regime factor. This type of endogenous impacts is not only sustained due to the autoregressive form of $w_{t}$, but also renders the transition probabilities time-varying because they are all functions of $\epsilon_{t}$ as will be shown subsequently. In the special case where $\epsilon_{t}$ and $v_{t+1}$ are orthogonal (i.e., $\rho=0_{n \times 1}$ ), the transition probabilities become constants and the model reduces to one with conventional Markov switching; in fact, there exists a one-to-one correspondence between our threshold-type switching specified by $(\alpha, \tau)$ and the Markov switching specified by two transition probabilities [see Chang et al. (2017), Lemma 2.1].

Since $p\left(v_{t+1} \mid \epsilon_{t}\right)$ is normal, we can replace $v_{t+1}$ by

$$
v_{t+1}=\sum_{k=1}^{n} \rho_{k} \epsilon_{k, t}+\left(1-\sum_{k=1}^{n} \rho_{k}^{2}\right)^{1 / 2} \eta_{t+1}, \quad \eta_{t+1} \sim \mathbb{N}(0,1)
$$

where $\left\{\epsilon_{k, t}\right\}_{k=1}^{n}$ and the idiosyncratic innovation $\eta_{t+1}$ are all orthogonal and have unit variance. On the surface, the residual $\eta_{t+1}$ of projecting $v_{t+1}$ onto $\epsilon_{t}$ appears to be a vague source of regime change in many economic applications where $\epsilon_{t}$ is interpreted as structural shocks with clear behavioral meanings. But it indeed captures potential misspecification of the transition equation - ideally one would expect the regime change to be fully driven by $\epsilon_{t}$ under the 'true' model - that leads to systematic disparities between model-implied and actual observables. To the extent that $\eta_{t+1}$ picks up those missing components beyond what are incorporated in $\epsilon_{t}$, we may readily call $\epsilon_{k, t}$ and $\eta_{t}$ the $k$-th internal and external innovation, respectively.

To quantify the importance of each source of regime change, iterate forward on (2.3) to obtain

\footnotetext{
${ }^{6}$ In the context of DSGE models, (2.2) represents a first-order approximation to the model's regime-specific policy function, and the coefficient and covariance matrices in (2.1)-(2.2) become sophisticated functions of structural parameters as well as the regime index. See Section 4 for such an example.
} 
$w_{t+h}=\alpha^{h} w_{t}+\sum_{j=1}^{h} \alpha^{h-j} v_{t+j}$ for $h \geqslant 1$. Combining with (2.5), we have the conditional variance

$$
\operatorname{Var}_{t}\left(w_{t+h}\right)=\sum_{k=1}^{n} \underbrace{\rho_{k}^{2} \sum_{j=1}^{h} \alpha^{2(h-j)}}_{\text {due to } \epsilon_{k}}+\underbrace{\left(1-\sum_{k=1}^{n} \rho_{k}^{2}\right) \sum_{j=1}^{h} \alpha^{2(h-j)}}_{\text {due to } \eta}=\underbrace{\sum_{j=1}^{h} \alpha^{2(h-j)}}_{\text {total }}, \quad h \geqslant 1
$$

It follows directly that the percent of the $h$-step-ahead forecast error variance of the regime factor due to the $k$-th internal (or external) innovation is given by $\rho_{k}^{2}$ (or $1-\sum_{k=1}^{n} \rho_{k}^{2}$ ), which is independent of $h$. Letting $h \rightarrow \infty, \rho_{k}^{2}$ (or $1-\sum_{k=1}^{n} \rho_{k}^{2}$ ) also measures the percentage contribution to the unconditional variance of the regime factor and hence the extent to which the $k$-th internal (or external) innovation contributes to the regime changes. For example, using a new Keynesian DSGE model with endogenous regime switching, Section 4 presents an empirical calculation on how much of the U.S. monetary policy shifts can be attributed to various internal innovations with distinct behavioral interpretations.

2.2 Filtering Algorithm Estimating the state space model (2.1)-(2.2) entails the dual objectives of likelihood evaluation and filtering, both of which require the calculation of integrals over the latent variables (i.e., $x_{t}$ and $s_{t}$ ). While the system is linear in $x_{t}$ and driven by Gaussian innovations, a complication arises from the presence of $s_{t}$; it introduces into the overall model structure additional nonlinearities that invalidate evaluating these integrals via the standard Kalman filter. Nevertheless, approximate analytical integration is still possible through a marginalization-collapsing procedure. In the marginalization step, we integrate out the state variables by exploiting the linear and Gaussian structure conditional on the most recent regime history, for which the standard Kalman filter can be applied. In the collapsing step, we approximate an otherwise exponentially growing number of history-dependent filtered distributions by two mixture Gaussian distributions in each period. This reduction effectively breaks the full history-dependence of the likelihood function and therefore makes the computation feasible and highly efficient. We call the resulting algorithm the endogenous-switching Kalman filter.

The key to operationalizing the above two-step procedure is an appropriate augmentation of the state space model. To that end, we introduce a dummy vector $d_{t}=\epsilon_{t}$ and augment the state vector 
$x_{t}$ as $\varsigma_{t}=\left[x_{t}^{\prime}, d_{t}^{\prime}\right]^{\prime}$. Accordingly, we rewrite the measurement and transition equations as

$$
\begin{gathered}
y_{t}=\underbrace{D_{s_{t}}+F_{s_{t}} z_{t}}_{\widetilde{D}_{s_{t}}}+\underbrace{\left(\begin{array}{cc}
Z_{s_{t}} & 0_{l \times n}
\end{array}\right)}_{\widetilde{Z}_{s_{t}}} \underbrace{\left(\begin{array}{c}
x_{t} \\
d_{t}
\end{array}\right)}_{\varsigma_{t}}+\Omega_{s_{t}}^{1 / 2} u_{t} \\
\underbrace{\left(\begin{array}{c}
x_{t} \\
d_{t}
\end{array}\right)}_{\varsigma_{t}}=\underbrace{\left(\begin{array}{c}
C_{s_{t}}+E_{s_{t}} z_{t} \\
0_{n \times 1}
\end{array}\right)}_{\widetilde{C}_{s_{t}}}+\underbrace{\left(\begin{array}{cc}
G_{s_{t}} & 0_{m \times n} \\
0_{n \times m} & 0_{n \times n}
\end{array}\right)}_{\widetilde{G}_{s_{t}}} \underbrace{\left(\begin{array}{c}
x_{t-1} \\
d_{t-1}
\end{array}\right)}_{s_{t-1}}+\underbrace{\left(\begin{array}{c}
M_{s_{t} \Sigma_{s_{t}}^{1 / 2}} \\
\mathrm{I}_{n}
\end{array}\right)}_{\widetilde{M}_{s_{t}}} \epsilon_{t}
\end{gathered}
$$

where the dependence of $\left(\widetilde{D}_{s_{t}}, \widetilde{C}_{s_{t}}\right)$ on $z_{t}$ has been suppressed for notational convenience. As will be shown in Algorithm 1 below, our main filtering algorithm, which is based on the augmented state space system (2.7)-(2.8), tracks the regime indices of both the current period and its preceding period in each recursion. At an exponentially rising computation cost though, one may improve the approximation by tracking even earlier regime history and, in the end, recover the exact likelihood function.

Let $\mathcal{F}_{t} \equiv \sigma\left(\left\{z_{s}, y_{s}\right\}_{s \leqslant t}\right)$ denote the information available at period $t$. Define the predictive probability of regime- $j$ at period $t$, joint with regime- $i$ at period $t-1$, as $p_{t \mid t-1}^{(i, j)} \equiv \mathbb{P}\left(s_{t-1}=i, s_{t}=j \mid \mathcal{F}_{t-1}\right)$ and the filtered marginal probability of regime- $j$ at period $t$ as $p_{t \mid t}^{j} \equiv \mathbb{P}\left(s_{t}=j \mid \mathcal{F}_{t}\right)$. Also write a battery of four conditional forecasts of $\varsigma_{t}$ and their forecast error covariances as

$$
\begin{aligned}
\varsigma_{t \mid t-1}^{(i, j)} & \equiv \mathbb{E}\left[\varsigma_{t} \mid s_{t-1}=i, s_{t}=j, \mathcal{F}_{t-1}\right] \\
P_{t \mid t-1}^{(i, j)} & \equiv \mathbb{E}\left[\left(\varsigma_{t}-\varsigma_{t \mid t-1}\right)\left(\varsigma_{t}-\varsigma_{t \mid t-1}\right)^{\prime} \mid s_{t-1}=i, s_{t}=j, \mathcal{F}_{t-1}\right]
\end{aligned}
$$

where $\varsigma_{t \mid t-1}=\mathbb{E}\left[\varsigma_{t} \mid \mathcal{F}_{t-1}\right]$. Then the filter can be summarized by the following steps.

\section{Algorithm 1. (Endogenous-Switching Kalman Filter)}

1. Initialization. For $i=0,1$, initialize the conditional mean vector and covariance matrix of $\varsigma_{0},\left(\varsigma_{0 \mid 0}^{i}, P_{0 \mid 0}^{i}\right)$, using the invariant distribution under regime- $i$. Set $p_{0 \mid 0}^{0}=\Phi\left(\tau \sqrt{1-\alpha^{2}}\right)$ and $p_{0 \mid 0}^{1}=1-p_{0 \mid 0}^{0}$ according to the invariant distribution of $w_{t}$, i.e., $\mathbb{N}\left(0,1 /\left(1-\alpha^{2}\right)\right)$.

2. Recursion. For $t=1, \ldots, T$, the filter accepts two sets of triple inputs $\left\{\left(\varsigma_{t-1 \mid t-1}^{i}, P_{t-1 \mid t-1}^{i}\right.\right.$, $\left.\left.p_{t-1 \mid t-1}^{i}\right)\right\}_{i=0}^{1}$, invokes the one-step Kalman filter to calculate the required integrals conditional on four possible mixes of the regimes in the current period and its preceding period, and returns two sets of updated triple outputs $\left\{\left(\varsigma_{t \mid t}^{j}, P_{t \mid t}^{j}, p_{t \mid t}^{j}\right)\right\}_{j=0}^{1}$.

(a) Forecasting. First, apply the forecasting step of the Kalman filter for the state variables 
to obtain

$$
\begin{aligned}
\varsigma_{t \mid t-1}^{(i, j)} & =\widetilde{C}_{j}+\widetilde{G}_{j} \varsigma_{t-1 \mid t-1}^{i} \\
P_{t \mid t-1}^{(i, j)} & =\widetilde{G}_{j} P_{t-1 \mid t-1}^{i} \widetilde{G}_{j}^{\prime}+\widetilde{M}_{j} \widetilde{M}_{j}^{\prime}
\end{aligned}
$$

for $i=0,1$ and $j=0,1$. Next, define $\lambda_{t} \equiv \rho^{\prime} \epsilon_{t}$ and compute the predictive joint probabilities

$$
\begin{aligned}
p_{t \mid t-1}^{(0,0)} & =\mathbb{P}\left(s_{t}=0 \mid s_{t-1}=0, \mathcal{F}_{t-1}\right) \mathbb{P}\left(s_{t-1}=0 \mid \mathcal{F}_{t-1}\right) \\
& =p_{t-1 \mid t-1}^{0} \int_{-\infty}^{\infty} \mathbb{P}\left(s_{t}=0 \mid s_{t-1}=0, \lambda_{t-1}, \mathcal{F}_{t-1}\right) p\left(\lambda_{t-1} \mid s_{t-1}=0, \mathcal{F}_{t-1}\right) d \lambda_{t-1}
\end{aligned}
$$

and $p_{t \mid t-1}^{(0,1)}=p_{t-1 \mid t-1}^{0}-p_{t \mid t-1}^{(0,0)}$. To evaluate the integral in (2.11), note that the predictive transition probability of remaining in regime- 0 between periods $t-1$ and $t$ can be computed as

$$
\begin{aligned}
\mathbb{P}\left(s_{t}=0 \mid s_{t-1}=0, \lambda_{t-1}, \mathcal{F}_{t-1}\right) & =\mathbb{P}\left(s_{t}=0 \mid s_{t-1}=0, \lambda_{t-1}\right) \\
& =\frac{\int_{-\infty}^{\tau \sqrt{1-\alpha^{2}}} \Phi_{\rho}\left(\tau-\alpha x / \sqrt{1-\alpha^{2}}-\lambda_{t-1}\right) p_{\mathbb{N}}(x \mid 0,1) d x}{\Phi\left(\tau \sqrt{1-\alpha^{2}}\right)}
\end{aligned}
$$

where $\Phi_{\rho}(x) \equiv \Phi\left(x / \sqrt{1-\rho^{\prime} \rho}\right)$, the first equality holds since $p\left(w_{t} \mid w_{t-1}, \lambda_{t-1}, \mathcal{F}_{t-1}\right)=$ $p\left(w_{t} \mid w_{t-1}, \lambda_{t-1}\right)$, and the second equality will be derived in Section 3.2. Clearly, the transition probability $\mathbb{P}\left(s_{t}=0 \mid s_{t-1}=0, \lambda_{t-1}\right)$ depends on the value of $\lambda_{t-1}$ and hence $\epsilon_{t-1}$ but becomes a constant when $\rho=0_{n \times 1}$. Moreover, we approximate

$$
p\left(\lambda_{t-1} \mid s_{t-1}=0, \mathcal{F}_{t-1}\right) \approx p_{\mathbb{N}}\left(\lambda_{t-1} \mid \rho^{\prime} \varsigma_{d, t-1 \mid t-1}^{0}, \rho^{\prime} P_{d, t-1 \mid t-1}^{0} \rho\right)
$$

where $\left(\varsigma_{d, t-1 \mid t-1}^{0}, P_{d, t-1 \mid t-1}^{0}\right)$ can be extracted from $\left(\varsigma_{t-1 \mid t-1}^{0}, P_{t-1 \mid t-1}^{0}\right)$ corresponding to $d_{t-1}$. To the extent that the filtered distribution of $\epsilon_{t-1}$ serves as an essential input into the approximation of $p\left(\lambda_{t-1} \mid s_{t-1}=0, \mathcal{F}_{t-1}\right)$, this justifies augmenting the state space system by the dummy vector $d_{t}=\epsilon_{t}$. Taken together, (2.11) can be approximated as

$$
p_{t \mid t-1}^{(0,0)} \approx \frac{p_{t-1 \mid t-1}^{0}}{\Phi\left(\tau \sqrt{1-\alpha^{2}}\right)} \int_{-\infty}^{\frac{\tau}{\sqrt{1-\rho^{\prime} \rho}}} \int_{-\infty}^{\tau \sqrt{1-\alpha^{2}}} p_{\mathbb{N}}\left(x, y \mid \mu_{0}, \Sigma_{0}\right) d x d y
$$


where

$$
\mu_{0} \equiv\left(\begin{array}{c}
0 \\
\frac{\rho^{\prime} \varsigma_{d, t-1 \mid t-1}^{0}}{\sqrt{1-\rho^{\prime} \rho}}
\end{array}\right), \quad \Sigma_{0} \equiv\left(\begin{array}{cc}
1 & \frac{\alpha}{\sqrt{1-\rho^{\prime} \rho} \sqrt{1-\alpha^{2}}} \\
\frac{\alpha}{\sqrt{1-\rho^{\prime} \rho} \sqrt{1-\alpha^{2}}} & 1+\frac{\alpha^{2}}{\left(1-\rho^{\prime} \rho\right)\left(1-\alpha^{2}\right)}+\frac{\rho^{\prime} P_{d, t-1 \mid t-1}^{0} \rho}{1-\rho^{\prime} \rho}
\end{array}\right)
$$

Similarly, we can approximate

$$
p_{t \mid t-1}^{(1,0)} \approx \frac{p_{t-1 \mid t-1}^{1}}{1-\Phi\left(\tau \sqrt{1-\alpha^{2}}\right)} \int_{-\infty}^{\frac{\tau}{\sqrt{1-\rho^{\prime} \rho}}} \int_{-\infty}^{-\tau \sqrt{1-\alpha^{2}}} p_{\mathbb{N}}\left(x, y \mid \mu_{1}, \Sigma_{1}\right) d x d y
$$

and $p_{t \mid t-1}^{(1,1)}=p_{t-1 \mid t-1}^{1}-p_{t \mid t-1}^{(1,0)}$, where

$$
\mu_{1} \equiv\left(\begin{array}{c}
0 \\
\frac{\rho^{\prime} \varsigma_{d, t-1 \mid t-1}^{1}}{\sqrt{1-\rho^{\prime} \rho}}
\end{array}\right), \quad \Sigma_{1} \equiv\left(\begin{array}{cc}
1 & \frac{-\alpha}{\sqrt{1-\rho^{\prime} \rho} \sqrt{1-\alpha^{2}}} \\
\frac{-\alpha}{\sqrt{1-\rho^{\prime} \rho} \sqrt{1-\alpha^{2}}} & 1+\frac{\alpha^{2}}{\left(1-\rho^{\prime} \rho\right)\left(1-\alpha^{2}\right)}+\frac{\rho^{\prime} P_{d, t-1 \mid t-1}^{1} \rho}{1-\rho^{\prime} \rho}
\end{array}\right)
$$

Finally, the integrals in (2.12)-(2.13) can be easily evaluated using the cumulative bivariate normal distribution function. Formulas for calculating all transition probabilities considered in this paper are in the Online Appendix.

(b) Likelihood evaluation. Apply the forecasting step of the Kalman filter for the observable variables to obtain

$$
\begin{aligned}
& y_{t \mid t-1}^{(i, j)}=\widetilde{D}_{j}+\widetilde{Z}_{j} \varsigma_{t \mid t-1}^{(i, j)} \\
& F_{t \mid t-1}^{(i, j)}=\widetilde{Z}_{j} P_{t \mid t-1}^{(i, j)} \widetilde{Z}_{j}^{\prime}+\Omega_{j}
\end{aligned}
$$

for $i=0,1$ and $j=0,1$. Then the period- $t$ likelihood contribution can be computed as

$$
p\left(y_{t} \mid \mathcal{F}_{t-1}\right)=\sum_{j=0}^{1} \sum_{i=0}^{1} p_{\mathbb{N}}\left(y_{t} \mid y_{t \mid t-1}^{(i, j)}, F_{t \mid t-1}^{(i, j)}\right) p_{t \mid t-1}^{(i, j)}
$$

(c) Filtering. First, apply the Bayes formula to update

$$
p_{t \mid t}^{(i, j)}=\frac{p_{\mathbb{N}}\left(y_{t} \mid y_{t \mid t-1}^{(i, j)}, F_{t \mid t-1}^{(i, j)}\right) p_{t \mid t-1}^{(i, j)}}{p\left(y_{t} \mid \mathcal{F}_{t-1}\right)}
$$

and calculate $p_{t \mid t}^{j}=\sum_{i=0}^{1} p_{t \mid t}^{(i, j)}$. Next, apply the filtering step of the Kalman filter for the state variables to obtain the updated conditional forecasts of $\varsigma_{t}$ and their forecast error 
covariances

$$
\begin{aligned}
\varsigma_{t \mid t}^{(i, j)} & =\varsigma_{t \mid t-1}^{(i, j)}+P_{t \mid t-1}^{(i, j)} \widetilde{Z}_{j}^{\prime}\left(F_{t \mid t-1}^{(i, j)}\right)^{-1}\left(y_{t}-y_{t \mid t-1}^{(i, j)}\right) \\
P_{t \mid t}^{(i, j)} & =P_{t \mid t-1}^{(i, j)}-P_{t \mid t-1}^{(i, j)} \widetilde{Z}_{j}^{\prime}\left(F_{t \mid t-1}^{(i, j)}\right)^{-1} \widetilde{Z}_{j} P_{t \mid t-1}^{(i, j)}
\end{aligned}
$$

for $i=0,1$ and $j=0,1$. To avoid a twofold increment in the number of cases to consider for the next period, collapse $\left(\varsigma_{t \mid t}^{(i, j)}, P_{t \mid t}^{(i, j)}\right)$ into $^{7}$

$$
\varsigma_{t \mid t}^{j} \approx \sum_{i=0}^{1} \frac{p_{t \mid t}^{(i, j)}}{p_{t \mid t}^{j}} \varsigma_{t \mid t}^{(i, j)}, \quad P_{t \mid t}^{j} \approx \sum_{i=0}^{1} \frac{p_{t \mid t}^{(i, j)}}{p_{t \mid t}^{j}}\left[P_{t \mid t}^{(i, j)}+\left(\varsigma_{t \mid t}^{j}-\varsigma_{t \mid t}^{(i, j)}\right)\left(\varsigma_{t \mid t}^{j}-\varsigma_{t \mid t}^{(i, j)}\right)^{\prime}\right]
$$

Further collapsing $\left(\varsigma_{t \mid t}^{j}, P_{t \mid t}^{j}\right)$ into

$$
\varsigma_{t \mid t} \approx \sum_{j=0}^{1} p_{t \mid t}^{j} \varsigma_{t \mid t}^{j}, \quad P_{t \mid t} \approx \sum_{j=0}^{1} p_{t \mid t}^{j}\left[P_{t \mid t}^{j}+\left(\varsigma_{t \mid t}-\varsigma_{t \mid t}^{j}\right)\left(\varsigma_{t \mid t}-\varsigma_{t \mid t}^{j}\right)^{\prime}\right]
$$

gives the filtered state variables.

3. Aggregation. The likelihood function is given by $p\left(Y_{1: T}\right)=\prod_{t=1}^{T} p\left(y_{t} \mid \mathcal{F}_{t-1}\right)$.

Several remarks about this filtering algorithm are in order. First, while its general structure resembles that of the mixture Kalman filter in Chen and Liu (2000), our filter requires no sequential Monte Carlo integration and is thus computationally efficient. By analytically integrating out $x_{t}$ and $s_{t}$, our filter also simplifies estimating the model via classical or Bayesian approach that would otherwise require a stochastic version of the expectation-maximization algorithm or Gibbs sampling, respectively [Wei and Tanner (1990), Tanner and Wong (1987)].

Second, in line with Kim (1994), the collapsing step (2.20) involves an approximation -its input $\varsigma_{t \mid t}^{(i, j)}$ does not calculate the conditional expectation $\mathbb{E}\left[\varsigma_{t} \mid s_{t-1}=i, s_{t}=j, \mathcal{F}_{t}\right]$ exactly since $p\left(\varsigma_{t} \mid s_{t-1}=\right.$ $\left.i, s_{t}=j, \mathcal{F}_{t}\right)$ amounts to a mixture of Gaussian distributions for $t>2$. Consequently, the period- $t$ likelihood $p\left(y_{t} \mid \mathcal{F}_{t-1}\right)$ and filtered states $\varsigma_{t \mid t}$ only approximately calculate their true values.

Third, an estimated regime factor $w_{t \mid t}$ can be easily extracted as a useful by-product of running the filter. Using the stored values of $\varsigma_{d, t-1 \mid t-1}^{i}, P_{d, t-1 \mid t-1}^{i}, p_{t-1 \mid t-1}^{i}, p_{\mathbb{N}}\left(y_{t} \mid y_{t \mid t-1}^{(i, j)}, F_{t \mid t-1}^{(i, j)}\right)$, and $p\left(y_{t} \mid \mathcal{F}_{t-1}\right)$,

${ }^{7}$ If $p_{t \mid t}^{j}=0$, the conditional probability $p_{t \mid t}^{(i, j)} / p_{t \mid t}^{j}=\mathbb{P}\left(s_{t-1}=i \mid s_{t}=j, \mathcal{F}_{t}\right)$ in $(2.20)$ is not well defined. In this case, we set $\left(\varsigma_{t \mid t}^{j}, P_{t \mid t}^{j}\right)=\left(\varsigma_{t \mid t}^{1-j}, P_{t \mid t}^{1-j}\right)$. 
it is straightforward to evaluate

$$
\begin{aligned}
p\left(w_{t} \mid \mathcal{F}_{t}\right) & =\sum_{i=0}^{1} \int_{-\infty}^{\infty} p\left(w_{t}, s_{t-1}=i, \lambda_{t-1} \mid \mathcal{F}_{t}\right) d \lambda_{t-1} \\
& =\sum_{i=0}^{1} \frac{p\left(y_{t} \mid w_{t}, s_{t-1}=i, \mathcal{F}_{t-1}\right) p_{t-1 \mid t-1}^{i}}{p\left(y_{t} \mid \mathcal{F}_{t-1}\right)} \int_{-\infty}^{\infty} p\left(w_{t} \mid s_{t-1}=i, \lambda_{t-1}\right) p\left(\lambda_{t-1} \mid \mathcal{F}_{t-1}\right) d \lambda_{t-1}
\end{aligned}
$$

where $p\left(y_{t} \mid w_{t}, s_{t-1}=i, \mathcal{F}_{t-1}\right)=p_{\mathbb{N}}\left(y_{t} \mid y_{t \mid t-1}^{(i, j)}, F_{t \mid t-1}^{(i, j)}\right)$ for $j=\mathbb{1}\left\{w_{t} \geqslant \tau\right\}$ and

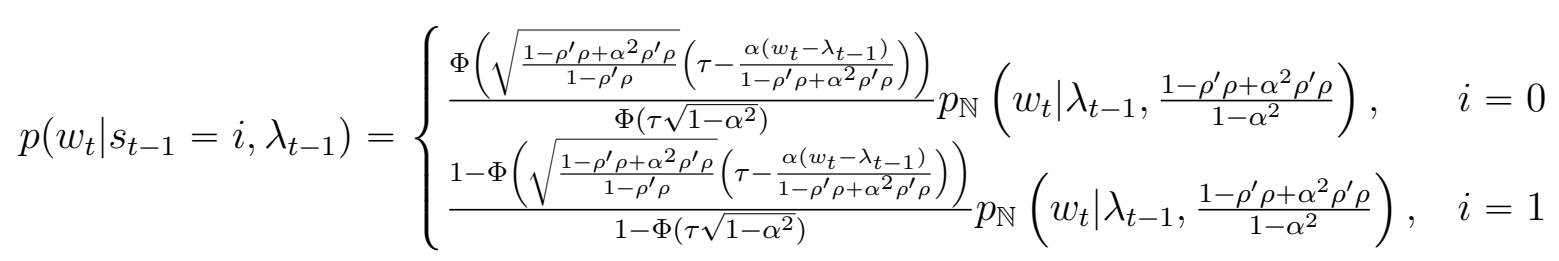

is derived in Corollary 3.3 of Chang et al. (2017). Moreover, we again approximate $p\left(\lambda_{t-1} \mid \mathcal{F}_{t-1}\right)$ by $p_{\mathbb{N}}\left(\lambda_{t-1} \mid \rho^{\prime} \varsigma_{d, t-1 \mid t-1}^{i}, \rho^{\prime} P_{d, t-1 \mid t-1}^{i} \rho\right)$ or simply the Dirac measure $\delta_{\rho^{\prime} \varsigma_{d, t-1 \mid t-1}^{i}}\left(\lambda_{t-1}\right)$ for $s_{t-1}=i$. Then the filtered regime factor can be computed as

$$
w_{t \mid t}=\int_{-\infty}^{\infty} w_{t} p\left(w_{t} \mid \mathcal{F}_{t}\right) d w_{t} \approx \sum_{k=1}^{N} w_{t}^{k} \hat{p}\left(w_{t}^{k} \mid \mathcal{F}_{t}\right)
$$

where we approximate $p\left(w_{t} \mid \mathcal{F}_{t}\right)$ by a discrete density function $\hat{p}\left(w_{t} \mid \mathcal{F}_{t}\right)$ defined on a swarm of grid points $\left\{w_{t}^{k}\right\}_{k=1}^{N}$ with their corresponding weights $\hat{p}\left(w_{t}^{k} \mid \mathcal{F}_{t}\right)=p\left(w_{t}^{k} \mid \mathcal{F}_{t}\right) / \sum_{k=1}^{N} p\left(w_{t}^{k} \mid \mathcal{F}_{t}\right)$.

Lastly, it is also possible to allow the model to switch among more than two regimes, but this would require introducing either multiple regime factors or threshold levels to operationalize in our setup. Such an extension, though theoretically appealing, is beyond the consideration of this paper. In the empirical application of Section 4, we extend our filter to estimate a monetary DSGE model with four possible regimes, where the endogenous policy regime is driven by an autoregressive latent factor and the exogenous volatility regime is driven by a Markov process.

2.3 DSGE Applichtion: The Road Ahead Over the past 20 years, DSGE models have become a useful tool for quantitative macroeconomic analysis in both academia and policymaking institutions. One particularly important development is the effort to incorporate the possibility of recurrent regime shifts (e.g., changes in monetary policy) into the model specification. Due to the substantial improvement in model fit, a multitude of empirical studies have proposed to estimate the state space representation of regime-switching DSGE models using likelihood-based econometric approaches [Schorfheide (2005), Liu et al. (2011), Bi and Traum (2012, 2014), Bianchi (2013), Davig and Doh (2014), Bianchi and Ilut (2017), Bianchi and Melosi (2017), Best and Hur (2019), among others]. 
We complement the recent literature on likelihood-based estimation of DSGE models with exogenous Markov switching by making regime change endogenous. At the core of our analysis is the endogenous feedback effect of underlying structural shocks on the regime generating process. As a result, economic agents update their beliefs each period about future regimes conditional on the realizations of shocks disturbing the economy. For pedagogical purposes, Section 3 employs a simple model adopted from Chang et al. (2018b) to endogenize regime switching in monetary policy, which admits analytical characterizations of the mechanism at work. Section 4 extends the simple model to a prototypical new Keynesian DSGE model, and derives its state space form that can be analyzed with our endogenous-switching Kalman filter introduced earlier in conjunction with a posterior sampler.

An important precursor to our study is Davig and Leeper (2006a), who applied the projection method to solve and calibrate a new Keynesian model where monetary policy rule changes whenever its target variables (e.g., inflation and output gap) cross some thresholds. ${ }^{8}$ More recently, Guerrieri and Iacoviello $(2015,2017)$ developed piecewise linear solution toolkit and likelihood-based estimation method for DSGE models subject to an occasionally binding constraint (e.g., the zero lower bound on nominal interest rates). In their setup, each state of the constraint - slack or binding - is handled as one of two distinct regimes under the same model. Like these studies, one may argue that it is more natural to assign the immediate triggers of regime switch to the state variables rather than the structural shocks. However, the dynamics of all state variables are ultimately driven by a small number of structural shocks. We therefore view the structural shocks that generate aggregate fluctuations as the macroeconomic origins of regime shifts, and establish a novel feedback channel by which they contribute to regime switching. Our analytical and empirical examples below illustrate how the underlying structural shocks impact agents' expectations formation and monetary policy regimes through this endogenous feedback channel.

We solve the model based on the assumption that economic agents, when forming rational expectations about future endogenous variables, exogenous shocks, and regime states, can observe their current and all past realizations. The regime factor, however, remains latent to agents as well as econometricians. ${ }^{9}$ As will be shown subsequently, it merely serves as an auxiliary variable that rationalizes the specific functional forms of time-varying transition probabilities. Consequently, the model can be solved without reference to the regime factor. From an empirical perspective, though,

\footnotetext{
${ }^{8}$ Using the same solution method, Bi and Traum $(2012,2014)$ estimated a real business cycle model where the government partially defaults on its debt whenever the debt level rises beyond a 'fiscal limit'.

${ }^{9}$ Allowing agents to observe the regime factor poses keen computational challenges to solving the model. Instead, we assume that agents always know which regime they are in. However, they need to compute the transition probabilities in order to make decisions. In the exogenous switching case, these probabilities are constant. Clearly, it is hard to believe that monetary policy would randomly switch between different states irrespective of the state of the economic system. In our model, these probabilities are a function of the history of shocks and the law of motion for the regime factor process.
} 
it is interesting to extract the latent regime factor from the data, which may be correlated with measured economic behavior in a meaningful way. For instance, using the same regime switching approach as in this paper, Chang et al. (2019) estimated a reduced-form model of monetary-fiscal regime changes and found that fiscal variables, particularly the tax to GDP ratio and net interest payment to government spending ratio, are among the most important variables in explaining the monetary regime factor.

\section{Analytical Example}

We first consider the simple frictionless model of inflation determination studied in Davig and Leeper (2006a), comprising a standard Fisher relation and an interest rate rule for monetary policy. In what follows, all variables are written in terms of log-deviations from their steady state values.

3.1 The Setup First, given a perfectly competitive endowment environment with flexible prices and one-period nominal bonds, the Fisher relation arises from the bond pricing equation and is, in its linearized form, given by

$$
i_{t}=\mathbb{E}_{t} \pi_{t+1}+\mathbb{E}_{t} r_{t+1}
$$

where $i_{t}$ denotes the short-term nominal interest rate, $\pi_{t}$ the inflation rate between periods $t-1$ and $t$, and $\mathbb{E}_{t}$ the conditional expectation given information available through period $t$. The real interest rate $r_{t}$ evolves as an autoregressive process

$$
r_{t}=\rho_{r} r_{t-1}+\sigma_{r} \epsilon_{r, t}, \quad \epsilon_{r, t} \sim \mathbb{N}(0,1)
$$

where $0 \leqslant \rho_{r}<1$ and $\sigma_{r}>0$.

Second, the monetary authority follows an interest rate feedback rule that systematically varies its response to contemporaneous inflation depending on the underlying policy regime, which is given by

$$
i_{t}=\phi_{s_{t}} \pi_{t}+\sigma_{i} \epsilon_{i, t}, \quad \phi_{s_{t}}=\phi_{0}\left(1-s_{t}\right)+\phi_{1} s_{t}, \quad \epsilon_{i, t} \sim \mathbb{N}(0,1)
$$

where $1<\phi_{0}<\phi_{1}$ and $\sigma_{i}>0 .{ }^{10}$ Here the response of policy rate to inflation is allowed to switch between, in the spirit of Leeper's (1991) terminology, 'more active' and 'less active' monetary regimes. The regime index evolves according to $s_{t}=\mathbb{1}\left\{w_{t} \geqslant \tau\right\}$ and the autoregressive regime factor follows $w_{t}=\alpha w_{t-1}+v_{t}$. In the case of $w_{t}=\pi_{t-1}$ and becomes observable, our model reduces to that of Davig and Leeper (2006a) where the monetary authority responds systematically more aggressively when lagged inflation exceeds a particular threshold, and less aggressively when it is

\footnotetext{
${ }^{10}$ For analytical tractability, we assume that the monetary authority does not respond to output gap.
} 
below the threshold.

Finally, we introduce an endogenous feedback channel from the current structural shocks to the future regime changes. There are two standardized shocks - the real rate shock $\epsilon_{r, t}$ and the monetary policy shock $\epsilon_{i, t}$-driving this simple economy, but for illustration purposes, we only consider the feedback from the current monetary policy shock $\epsilon_{i, t}$ through its potential correlation with the next period regime factor innovation $v_{t+1}$. That is,

$$
\left(\begin{array}{c}
\epsilon_{i, t} \\
v_{t+1}
\end{array}\right) \sim \mathbb{N}\left(\left(\begin{array}{l}
0 \\
0
\end{array}\right),\left(\begin{array}{cc}
1 & \rho \\
\rho & 1
\end{array}\right)\right), \quad-1<\rho<1
$$

where $\rho=\operatorname{corr}\left(\epsilon_{i, t}, v_{t+1}\right)$ is a correlation parameter that measures the strength of endogeneity in regime switching. The above specification is simple enough to admit an analytical solution, yet rich enough to highlight the general features of a rational expectations model with endogenous regime change in monetary policy.

It follows from (3.4) that

$$
w_{t+1}=\alpha w_{t}+\rho \epsilon_{i, t}+\sqrt{1-\rho^{2}} \eta_{t+1}, \quad \eta_{t+1} \sim \mathbb{N}(0,1)
$$

where the internal innovation $\epsilon_{i, t}$ and the external innovation $\eta_{t+1}$ are orthogonal to each other. This alternative representation of the regime factor points to the endogenous feedback from monetary interventions to the regime generating process. For example, when $\epsilon_{i, t}$ and $v_{t+1}$ are orthogonal (i.e., $\rho=0$ ), regime shifts become the outcome of an exogenous process driven entirely by the non-structural shock $\eta_{t+1}$; as $\rho$ approaches to one in absolute value, today's monetary shocks bear more directly on tomorrow's regime factor; when $|\rho|=1$, future regimes turn out to depend only on monetary shock in the current period. In general, one would expect $0<|\rho|<1 .{ }^{11}$ The autoregressive coefficient $\alpha$, on the other hand, determines the persistency and hence the expected duration of each regime as $\alpha$ takes values towards positive (negative) unity, the model will on average undergo less (more) frequent regime shifts.

3.2 Transition Probability Like any regime switching model, it is essential to compute the associated transition probabilities. From a modeling point of view, it can be helpful to treat the regime factor as a computational device that produces the specific functional forms of transition probabilities adopted by this paper. To see that, first note

$$
\mathbb{P}\left(w_{t+1}<\tau \mid w_{t}, \epsilon_{i, t}\right)=\mathbb{P}\left(\eta_{t+1}<\frac{\tau-\alpha w_{t}-\rho \epsilon_{i, t}}{\sqrt{1-\rho^{2}}} \mid w_{t}, \epsilon_{i, t}\right)=\Phi_{\rho}\left(\tau-\alpha w_{t}-\rho \epsilon_{i, t}\right)
$$

\footnotetext{
${ }^{11}$ From now on, we dispense with $|\rho|=1$ because predetermined regimes, though theoretically possible, make less economic sense.
} 
where $\Phi_{\rho}(x)=\Phi\left(x / \sqrt{1-\rho^{2}}\right)$. Moreover, $w_{t}$ is independent of $\epsilon_{i, t}$ and follows $\mathbb{N}\left(0,1 /\left(1-\alpha^{2}\right)\right)$. Therefore, we can obtain the transition probability of staying in regime-0 (i.e., the less active regime) between periods $t$ and $t+1$ explicitly as

$$
\begin{aligned}
p_{00}\left(\epsilon_{i, t}\right) & =\mathbb{P}\left(s_{t+1}=0 \mid s_{t}=0, \epsilon_{i, t}\right) \\
& =\frac{\mathbb{P}\left(w_{t+1}<\tau, w_{t}<\tau \mid \epsilon_{i, t}\right)}{\mathbb{P}\left(w_{t}<\tau\right)} \\
& =\frac{\int_{-\infty}^{\tau \sqrt{1-\alpha^{2}}} \Phi_{\rho}\left(\tau-\alpha x / \sqrt{1-\alpha^{2}}-\rho \epsilon_{i, t}\right) p_{\mathbb{N}}(x \mid 0,1) d x}{\Phi\left(\tau \sqrt{1-\alpha^{2}}\right)} \\
& =\frac{\int_{-\infty}^{\frac{\tau-\rho \epsilon_{i, t}}{\sqrt{1-\rho^{2}}}} \int_{-\infty}^{\tau \sqrt{1-\alpha^{2}}} p_{\mathbb{N}}\left(x, y \mid \mu_{0}, \Sigma_{0}\right) d x d y}{\Phi\left(\tau \sqrt{1-\alpha^{2}}\right)}
\end{aligned}
$$

where $\mu_{0}=[0,0]^{\prime}, \Sigma_{0}=\left[1, c ; c, 1+c^{2}\right]$, and $c=\alpha /\left(\sqrt{1-\rho^{2}} \sqrt{1-\alpha^{2}}\right)$. Analogously, the transition probability from regime-1 (i.e., the more active regime) in period $t$ to regime- 0 in period $t+1$ can be computed as

$$
\begin{aligned}
p_{10}\left(\epsilon_{i, t}\right) & =\mathbb{P}\left(s_{t+1}=0 \mid s_{t}=1, \epsilon_{i, t}\right) \\
& =\frac{\mathbb{P}\left(w_{t+1}<\tau, w_{t} \geqslant \tau \mid \epsilon_{i, t}\right)}{\mathbb{P}\left(w_{t} \geqslant \tau\right)} \\
& =\frac{\int_{\tau \sqrt{1-\alpha^{2}}}^{\infty} \Phi_{\rho}\left(\tau-\alpha x / \sqrt{1-\alpha^{2}}-\rho \epsilon_{i, t}\right) p_{\mathbb{N}}(x \mid 0,1) d x}{1-\Phi\left(\tau \sqrt{1-\alpha^{2}}\right)} \\
& =\frac{\int_{-\infty}^{\frac{\tau-\rho \epsilon_{i, t}}{\sqrt{1-\rho^{2}}}} \int_{-\infty}^{-\tau \sqrt{1-\alpha^{2}}} p_{\mathbb{N}}\left(x, y \mid \mu_{1}, \Sigma_{1}\right) d x d y}{1-\Phi\left(\tau \sqrt{1-\alpha^{2}}\right)}
\end{aligned}
$$

where $\mu_{1}=[0,0]^{\prime}$ and $\Sigma_{1}=\left[1,-c ;-c, 1+c^{2}\right]$. Accordingly, we have $p_{01}\left(\epsilon_{i, t}\right)=1-p_{00}\left(\epsilon_{i, t}\right)$ and $p_{11}\left(\epsilon_{i, t}\right)=1-p_{10}\left(\epsilon_{i, t}\right)$. Finally, the integrals in (3.6)-(3.7) can be easily evaluated using the cumulative bivariate normal distribution function. See the Online Appendix for derivation details.

In sum, our endogenous feedback mechanism renders the transition probabilities, which now become an integral part of the model solution, time-varying because they are all functions of $\epsilon_{i, t}$. A key difference from Chang et al. (2017), though, lies in that their $\epsilon_{i, t}$ corresponds to a univariate regression error that can be readily computed given the data and parameter values, whereas ours represents structural shocks whose values remain latent to the econometrician and hence must be inferred from the data. In the special case of $\rho=0$, transition probabilities (3.6)-(3.7) become constants and our model reduces to one with conventional Markov switching.

Figure 2 plots transition probabilities as functions of monetary policy shock at selected values of $(\alpha, \tau, \rho)$, which is intended to highlight the distinct role of each parameter in shaping these functions while holding other parameters fixed. Overall, $(\alpha, \tau)$ uniquely determine the constant levels of 

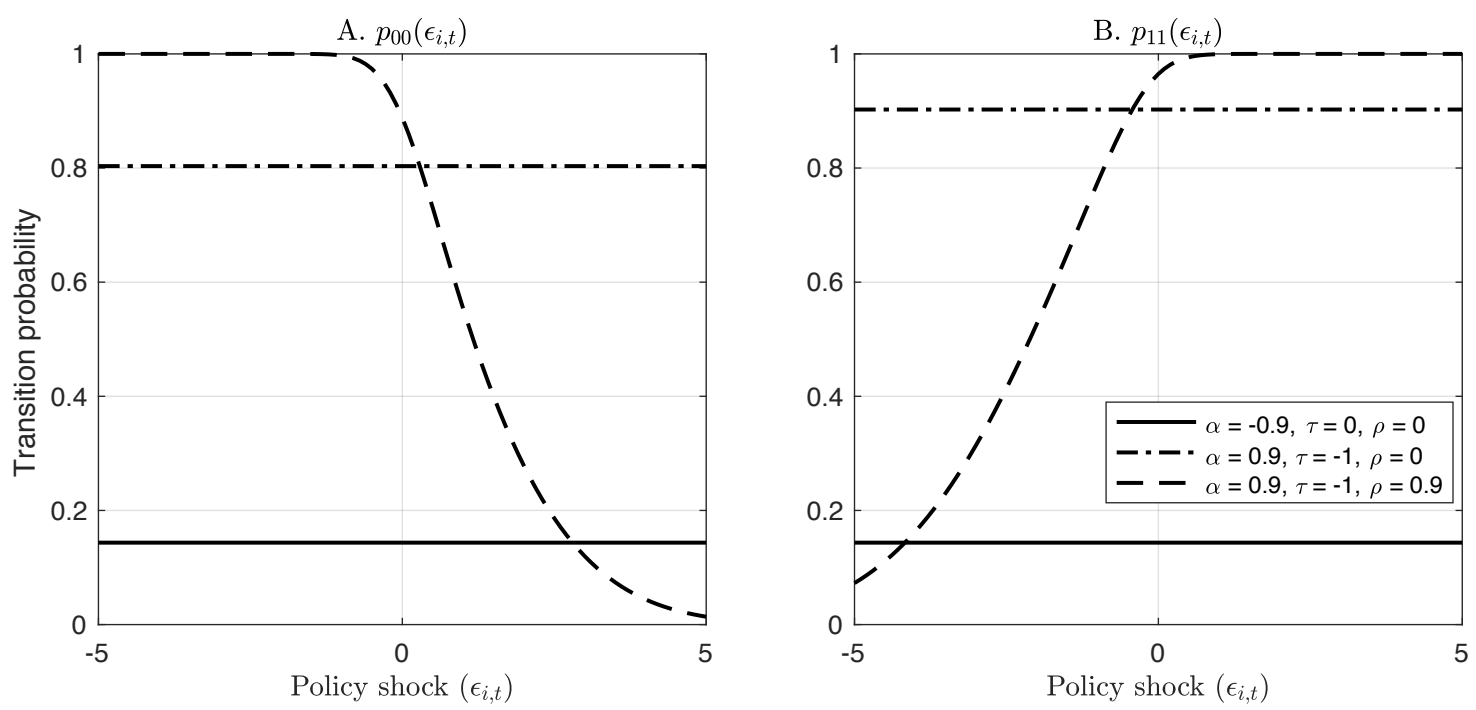

Figure 2: Transition probabilities as functions of $\epsilon_{i, t}$ at selected values of $(\alpha, \tau, \rho)$.

$\left(p_{00}, p_{11}\right)$ under exogenous Markov switching (solid and dash-dotted lines) owing to their one-to-one correspondence. Specifically, increasing the value of $\alpha$ from -0.9 to 0.9 , for example, raises the likelihood of remaining in the current regime by making the regime factor more persistent. Meanwhile, decreasing the value of $\tau$ from 0 to -1 favors the more active regime by making it relatively easier for the regime factor to stay above the threshold. On the other hand, the endogeneity parameter $\rho$ introduces shock-specific variations into $\left(p_{00}, p_{11}\right)$ (dashed line). Due to the positive feedback effect $(\rho=0.9)$, for instance, a one-time unanticipated tightening $\epsilon_{i, t}>0$ (loosening $\epsilon_{i, t}<0$ ) of policy today increases the probability of staying in or shifting to the systematically tighter (looser) policy in the next period. Of course, the overall shapes of transition probability functions rest on all three parameters.

3.3 Equilibrium Characteristics Together with the transition probabilities (3.6)-(3.7), equations (3.1)-(3.3) constitute a nonlinear rational expectations system in the endogenous variables $\left(\pi_{t}, i_{t}\right)$ that is driven by the exogenous variables $\left(r_{t}, \epsilon_{i, t}\right)$. Substituting (3.2) and (3.3) into (3.1) delivers a regime-specific expectational difference equation in inflation

$$
\phi_{s_{t}} \pi_{t}+\sigma_{i} \epsilon_{i, t}=\mathbb{E}_{t} \pi_{t+1}+\rho_{r} r_{t}
$$

Now solving the model entails mapping the minimum set of state variables $\left(r_{t}, \epsilon_{i, t}\right)$ into the endogenous variable $\left(\pi_{t}\right)$. We find such a minimum state variable solution with the method of undetermined coefficients by postulating a regime-specific solution that takes an additive form

$$
\pi_{t}=A_{s_{t}} r_{t}+B_{s_{t}} \epsilon_{i, t}
$$


Chang et al. (2018b) show that these coefficients can be expressed as

$$
A_{s_{t}}=\frac{\rho_{r}}{\phi_{s_{t}}} \frac{\left(\phi_{1}-\phi_{0}\right) p_{s_{t}, 0}\left(\epsilon_{i, t}\right)+\phi_{1}\left(\frac{\phi_{0}}{\rho_{r}}-\mathbb{E} p_{00}\left(\epsilon_{i, t}\right)\right)+\phi_{0} \mathbb{E} p_{10}\left(\epsilon_{i, t}\right)}{\left(\phi_{1}-\rho_{r}\right)\left(\frac{\phi_{0}}{\rho_{r}}-\mathbb{E} p_{00}\left(\epsilon_{i, t}\right)\right)+\left(\phi_{0}-\rho_{r}\right) \mathbb{E} p_{10}\left(\epsilon_{i, t}\right)}, \quad B_{s_{t}}=-\frac{\sigma_{i}}{\phi_{s_{t}}}
$$

where $A_{s_{t}}$ also depends on $\epsilon_{i, t}$ and the unconditional expectations $\mathbb{E} p_{00}\left(\epsilon_{i, t}\right)$ and $\mathbb{E} p_{10}\left(\epsilon_{i, t}\right)$ are constant terms.

Two special cases arise from the general solution (3.10). When regime changes are purely exogenous (i.e., $\rho=0)$, (3.10) reduces to

$$
A_{s_{t}}=\frac{\rho_{r}}{\phi_{s_{t}}} \frac{\left(\phi_{1}-\phi_{0}\right) p_{s_{t}, 0}+\phi_{1}\left(\frac{\phi_{0}}{\rho_{r}}-p_{00}\right)+\phi_{0} p_{10}}{\left(\phi_{1}-\rho_{r}\right)\left(\frac{\phi_{0}}{\rho_{r}}-p_{00}\right)+\left(\phi_{0}-\rho_{r}\right) p_{10}}, \quad B_{s_{t}}=-\frac{\sigma_{i}}{\phi_{s_{t}}}
$$

where $p_{s_{t}, 0}$ and hence $A_{s_{t}}$ become independent of $\epsilon_{i, t}$. Further imposing the restriction $\phi_{0}=\phi_{1}=$ $\phi>1$ gives the equilibrium inflation under fixed regime

$$
A_{s_{t}}=\frac{\rho_{r}}{\phi-\rho_{r}}, \quad B_{s_{t}}=-\frac{\sigma_{i}}{\phi}
$$

where both $A_{s_{t}}$ and $B_{s_{t}}$ are non-random constants. It is clear from (3.12) that a more aggressive monetary stance (i.e., higher $\phi$ ) can effectively insulate inflation against exogenous disturbances.

In all cases, we have $A_{s_{t}}>0$ so that a positive real rate shock, as it does in a fixed regime, raises the contemporaneous demand for consumption and thus inflation. With endogenous feedback in regime change, it immediately follows from (3.10) that two distinct effects on inflation emerge after a monetary policy intervention. First, conditioning on the prevailing policy regime, a monetary contraction tends to curtail inflation through its linear and direct effect captured by the $B_{s_{t}}<0$ term. Second, and more importantly, a monetary contraction also generates an endogenous expectations-formation effect - the difference between the impacts of a shock when the regime switches endogenously and when it switches exogenously - that is captured by the transition probability $p_{s_{t}, 0}\left(\epsilon_{i, t}\right)$ in the $A_{s_{t}}$ term. This nonlinear effect arises in that the intervention induces a change in agents' beliefs about the future policy regime. The resultant adjustment in agents' behavior can shift the projected path and probability distribution of equilibrium inflation in economically meaningful ways. As opposed to the endogenous switching case, such forward-looking effect vanishes in (3.11) under exogenous switching and thus there is no channel by which monetary interventions can alter agents' expectations about future regime.

To make the analytics more concrete, Figure 3 isolates the endogenous expectations-formation effect by comparing the contemporaneous responses of inflation to exogenous shocks under endogenous $(\rho=0.9)$ and exogenous switching $(\rho=0)$. We consider a policy process that adjusts nominal 

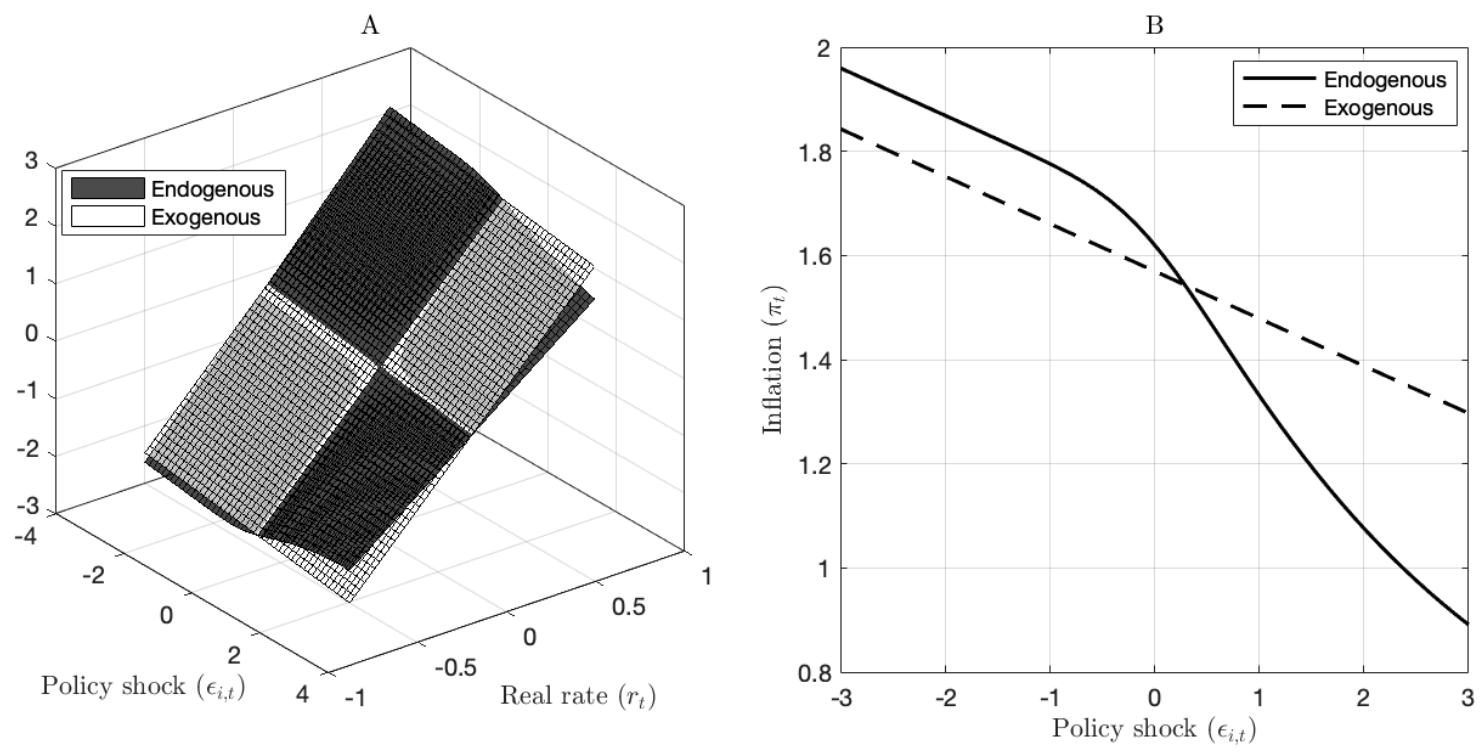

Figure 3: Impulse response functions for inflation. Notes: Parameter settings under endogenous switching are $\left(\phi_{0}, \phi_{1}\right)=(1.1,1.5),\left(\rho_{r}, \sigma_{r}, \sigma_{i}\right)=(0.9,0.1,0.1)$, and $(\alpha, \tau, \rho)=(0.9,-1,0.9)$. Responses under exogenous switching are obtained by setting $\rho=0$ while keeping other parameters unchanged. The current regime is set to be less active, i.e., $s_{t}=0$.

rate only 'mildly' in the less active regime $\left(\phi_{0}=1.1\right)$, but to a degree more consistent with the standard Taylor rule specification when the more active regime is in place $\left(\phi_{1}=1.5\right)$. We also have the regime factor relatively persistent $(\alpha=0.9)$ and somewhat favor the more active regime $(\tau=-1) .{ }^{12}$ Panel B illustrates a slice of the impulse response surface reported in Panel A for a given positive real rate $r_{t}=0.5 \%$. Starting with the less active policy, contractionary monetary shocks trigger a positive feedback effect with endogenous switching, which leads agents to revise their beliefs towards a tigher policy in the subsequent period (as evinced by Figure 2). In comparison with the exogenous switching case, this shift in expectations about future policy helps to further mitigate the inflationary effect of a positive real rate on impact. Analogously, expansionary monetary shocks trigger a negative feedback effect that bolsters agents' beliefs in the looser policy for the next period. Although less noticeable, the same positive real rate thus has larger impacts on current inflation. ${ }^{13}$

Formally, we measure expectations-formation effects from a policy intervention based on conditional inflation forecasts along the lines of Leeper and Zha (2003). Since private agents can observe current and all past realizations of endogenous variables $\left(\pi_{t}, i_{t}\right)$, exogenous shocks $\left(r_{t}, \epsilon_{i, t}\right)$, and regime states $s_{t}$, they formulate rational expectations about future inflation based on the information set $\mathcal{F}_{T}=\sigma\left(\left\{\pi_{t}, i_{t}, r_{t}, \epsilon_{i, t}, s_{t}\right\}_{t=0}^{T}\right)$. Let $I_{T}$ be a hypothetical intervention at period $T$, specified

\footnotetext{
${ }^{12}$ The implied transition probabilities under exogenous switching are given by $\left(p_{00}, p_{11}\right)=(0.8,0.9)$. See Figure 2 for a visualization of the transition probability functions associated with endogenous switching.

${ }^{13}$ The analysis with a negative real rate is similar and therefore omitted here to conserve space.
} 

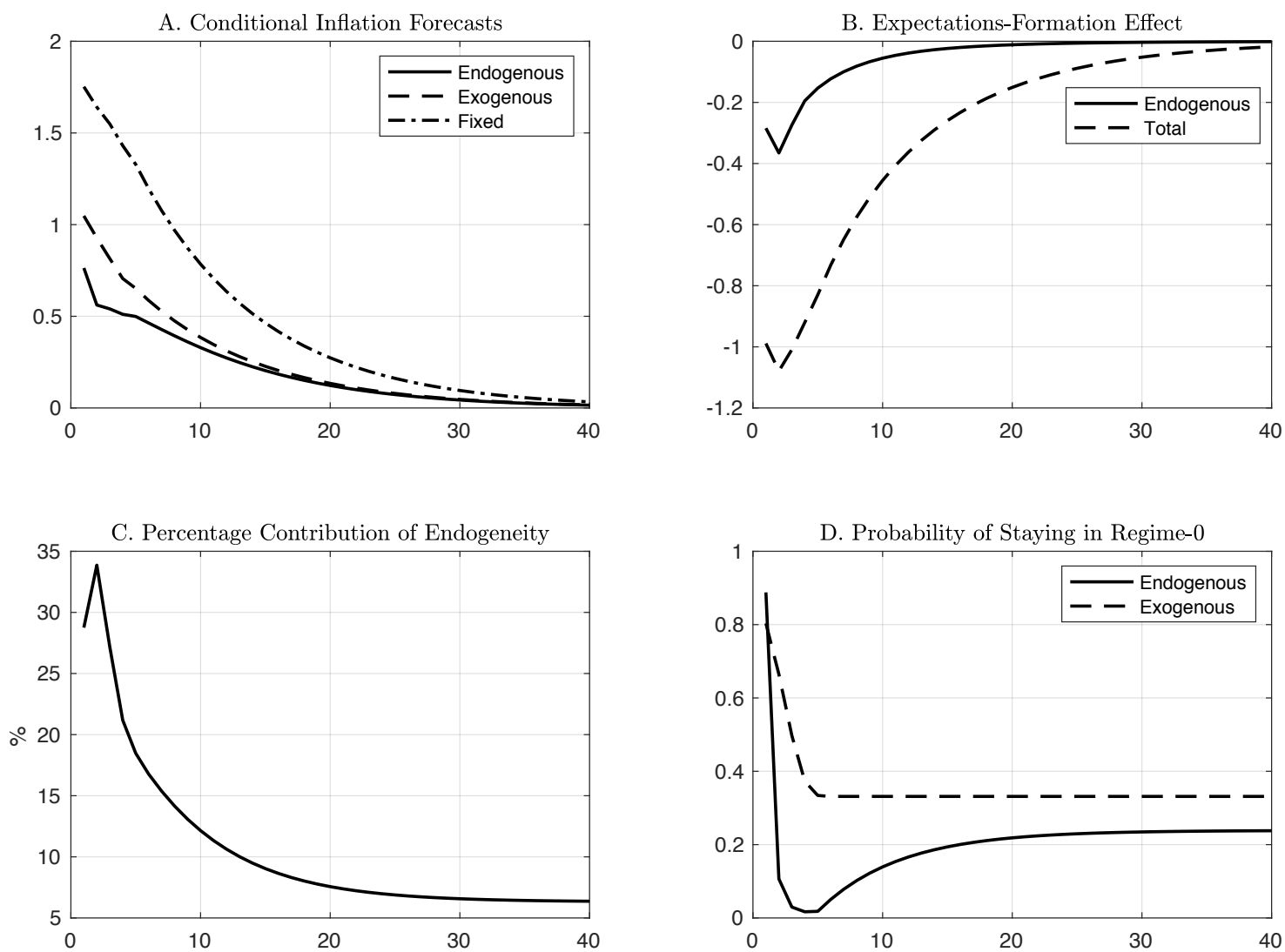

Figure 4: Expectations-formation effects on inflation. Notes: Panel A plots the inflation forecasts conditional on the intervention $I_{T}=\{3,2,1,0.5,0, \ldots, 0\}$. Panel B plots their differentials as in the definitions of expectations-formation effects. Panel $\mathrm{C}$ reports the ratio of endogenous to total effects. Panel D plots the $K$-period-ahead probabilities of remaining in the less active regime. The current set of state variables is set to $\left(r_{T}, \epsilon_{i, T}, s_{T}\right)=(0.5 \%, 0,0)$. See Figure 3 notes for parameter settings.

as a $K$-period sequence of exogenous policy actions $I_{T}=\left\{\epsilon_{i, T+1}, \ldots, \epsilon_{i, T+K}\right\}$. Given the analytical solution under endogenous switching (3.10), it is straightforward to evaluate the forecast of $\pi_{T+K}$ conditional on $I_{T}$ as

$$
\mathbb{E}\left[\pi_{T+K} \mid I_{T}, \mathcal{F}_{T}\right]=\left(p_{s_{T}, 0} A_{0}\left(\epsilon_{i, T+K}\right)+p_{s_{T}, 1} A_{1}\left(\epsilon_{i, T+K}\right)\right) \rho_{r}^{K} r_{T}+\left(p_{s_{T}, 0} B_{0}+p_{s_{T}, 1} B_{1}\right) \epsilon_{i, T+K}
$$

where $p_{s_{T}, s_{T+K}}$ for $s_{T+K}=0,1$ are the $K$-period-ahead transition probabilities that depend on the sequence $\left\{\epsilon_{i, T}, \ldots, \epsilon_{i, T+K-1}\right\}$. Likewise, forecasts under exogenous switching $\mathbb{E}\left[\pi_{T+K} \mid I_{T}, \mathcal{F}_{T}, \rho=0\right]$ and fixed regime $\mathbb{E}\left[\pi_{T+K} \mid I_{T}, \mathcal{F}_{T}, s_{t}=0, t=T+1, \ldots, T+K\right]$ can be computed using solutions (3.11) and (3.12), respectively. The total expectations-formation effect - a term coined by Leeper and Zha (2003) - refers to the difference between the impacts of a policy intervention when regime can and cannot switch, respectively

$$
\text { Total effect } \equiv \mathbb{E}\left[\pi_{T+K} \mid I_{T}, \mathcal{F}_{T}\right]-\mathbb{E}\left[\pi_{T+K} \mid I_{T}, \mathcal{F}_{T}, s_{t}=0, t=T+1, \ldots, T+K\right]
$$


We focus on its endogenous component which is more relevant in our context

$$
\text { Endogenous effect } \equiv \mathbb{E}\left[\pi_{T+K} \mid I_{T}, \mathcal{F}_{T}\right]-\mathbb{E}\left[\pi_{T+K} \mid I_{T}, \mathcal{F}_{T}, \rho=0\right]
$$

Of course, the difference between (3.13) and (3.14) quantifies the exogenous component.

For illustration purpose, we suppose the monetary authority undertakes a one-year contractionary intervention $I_{T}=\{3,2,1,0.5,0, \ldots, 0\}$ with gradually declining magnitudes from 3 to $1 / 2$ standard deviations $\sigma_{i}$ of the monetary policy shock. Panel A of Figure 4 records the conditional forecasts of inflation obtained from the three models. ${ }^{14}$ As expected, the intervention further tempers the initial inflationary impact of $r_{T}=0.5 \%$ by about $1 \%$ with endogenous switching and $0.7 \%$ with exogenous switching relative to the fixed regime baseline. These discrepancies among the forecasts translate into sizable expectations-formation effects in Panel B, which slowly taper off over the 10year forecasting horizon. More importantly, over $30 \%$ of the total effect during the intervention can be attributed to its endogenous component (see Panel C). At very short horizons, the consecutive spikes in policy rate put forth a significantly positive feedback effect with endogenous switching, placing nearly all probability weight on the more active regime (see Panel D). Once the intervention ends after one year, the probability of remaining in the less active regime eventually reverts to a permanently lower level relative to the exogenous switching case.

\section{Empirical Illustration}

We now consider the small-scale new Keynesian DSGE model presented in An and Schorfheide (2007) with the following features: a representative household and a continuum of monopolistically competitive firms; each firm produces a differentiated good and faces nominal rigidity in terms of quadratic price adjustment cost; a cashless economy with one-period nominal bonds; a monetary authority that controls nominal interest rate as well as a fiscal authority that passively adjusts lump-sum taxes to ensure its budgetary solvency; a labor-augmenting technology that induces a stochastic trend in consumption and output.

4.1 The Setup The model's equilibrium conditions in terms of the detrended variables can be summarized as follows. First, the household's optimizing behavior implies

$$
1=\beta \mathbb{E}_{t}\left[\left(\frac{c_{t+1}}{c_{t}}\right)^{-\tau_{c}} \frac{R_{t}}{\gamma z_{t+1} \pi_{t+1}}\right]
$$

where $0<\beta<1$ is the discount factor, $\tau_{c}>0$ the coefficient of relative risk aversion, $c_{t}$ the detrended consumption, $R_{t}$ the nominal interest rate, $\pi_{t}$ the inflation between periods $t-1$ and $t$,

\footnotetext{
${ }^{14}$ In the special case of one-period intervention $I_{T}=\{1,0, \ldots, 0\}$, these forecasts correspond to the conventional impulse response analysis.
} 
$z_{t}$ an exogenous shock to the labor-augmenting technology that grows on average at the rate $\gamma$, and $\mathbb{E}_{t}$ represents the conditional expectation given information available at time $t$. The firm's optimal price-setting behavior yields

$$
1=\frac{1-c_{t}^{\tau_{c}}}{\nu}+\phi\left(\pi_{t}-\pi\right)\left[\left(1-\frac{1}{2 \nu}\right) \pi_{t}+\frac{\pi}{2 \nu}\right]-\phi \beta \mathbb{E}_{t}\left[\left(\frac{c_{t+1}}{c_{t}}\right)^{-\tau_{c}} \frac{y_{t+1}}{y_{t}}\left(\pi_{t+1}-\pi\right) \pi_{t+1}\right]
$$

where $1 / \nu>1$ is the elasticity of demand for each differentiated good, $\phi$ the degree of price stickiness that relates to the slope of the so-called new Keynesian Phillips curve $\kappa$ via $\phi=\tau_{c}(1-\nu) /\left(\nu \pi^{2} \kappa\right)$, $\pi$ the steady state inflation, and $y_{t}$ the detrended output. The goods market clearing condition is given by

$$
y_{t}=c_{t}+\left(1-\frac{1}{g_{t}}\right) y_{t}+\frac{\phi}{2}\left(\pi_{t}-\pi\right)^{2} y_{t}
$$

where $g_{t}$ is an exogenous government spending shock with the steady state $g$.

Second, the monetary authority follows an interest rate feedback rule that reacts to deviations of inflation from its steady state and output from its potential value

$$
R_{t}=R_{t}^{* 1-\rho_{R}} R_{t-1}^{\rho_{R}} e^{\sigma_{R} \epsilon_{R, t}}, \quad R_{t}^{*}=R\left(\frac{\pi_{t}}{\pi}\right)^{\psi_{\pi}}\left(\frac{y_{t}}{y_{t}^{*}}\right)^{\psi_{y}}
$$

where $0 \leqslant \rho_{R}<1$ is the degree of interest rate smoothing, $\sigma_{R}>0, R$ the steady state nominal interest rate, $\psi_{\pi}>0$ and $\psi_{y}>0$ the policy rate responsive coefficients, $y_{t}^{*}=(1-\nu)^{1 / \tau_{c}} g_{t}$ the detrended potential output that would prevail in the absence of nominal rigidities (i.e., $\phi=0$ ), and $\epsilon_{R, t}$ an exogenous policy shock.

Finally, both $\ln z_{t}$ and $\ln g_{t}$ evolve as autoregressive processes

$$
\begin{aligned}
& \ln z_{t}=\rho_{z} \ln z_{t-1}+\sigma_{z} \epsilon_{z, t} \\
& \ln g_{t}=\left(1-\rho_{g}\right) \ln g+\rho_{g} \ln g_{t-1}+\sigma_{g} \epsilon_{g, t}
\end{aligned}
$$

where $0 \leqslant \rho_{z}, \rho_{g}<1$ and $\sigma_{z}, \sigma_{g}>0$. The model is driven by the three innovations $\left(\epsilon_{z, t}, \epsilon_{g, t}, \epsilon_{R, t}\right)$ that are serially uncorrelated, independent of each other at all leads and lags, and normally distributed with zero mean and unit standard deviation.

There has been ample empirical evidence of time variation in estimated monetary policy rules documented in the literature. To keep the illustration simple and concrete, we allow the response of policy rate to inflation deviations to switch between more active and less active (or possibly 'passive') monetary policy regimes

$$
\psi_{\pi}\left(s_{t}^{\mathrm{Pol}}\right)=\psi_{\pi, 0}\left(1-s_{t}^{\mathrm{Pol}}\right)+\psi_{\pi, 1} s_{t}^{\mathrm{Pol}}, \quad 0 \leqslant \psi_{\pi, 0}<\psi_{\pi, 1}
$$


where the policy regime index evolves according to $s_{t}^{\mathrm{Pol}}=\mathbb{1}\left\{w_{t} \geqslant \tau\right\}$ and the regime factor follows $w_{t}=\alpha w_{t-1}+v_{t}$. To introduce the sources of endogeneity in regime change, we allow all current structural shocks to jointly influence the next period regime through their correlations with the innovation $v_{t+1}$. That is,

$$
\left(\begin{array}{c}
\epsilon_{t} \\
v_{t+1}
\end{array}\right) \sim \mathbb{N}\left(\left(\begin{array}{c}
0_{3 \times 1} \\
0
\end{array}\right),\left(\begin{array}{cc}
\mathrm{I}_{3} & \rho \\
\rho^{\prime} & 1
\end{array}\right)\right), \quad \rho^{\prime} \rho<1
$$

where $\rho=\left[\rho_{z v}, \rho_{g v}, \rho_{R v}\right]^{\prime}=\operatorname{corr}\left(\epsilon_{t}, v_{t+1}\right)$. We also allow the standard deviation of each shock to switch between high volatility and low volatility

$$
\sigma_{i}\left(s_{t}^{\mathrm{Vol}}\right)=\sigma_{i, 0}\left(1-s_{t}^{\mathrm{Vol}}\right)+\sigma_{i, 1} s_{t}^{\mathrm{Vol}}, \quad 0<\sigma_{i, 0}<\sigma_{i, 1}, \quad i \in\{z, g, R\}
$$

where the volatility regime index $s_{t}^{\text {Vol }}$ follows a Markov chain defined by two states $\{0,1\}$ and transition probabilities $p_{i j}, i, j=0,1$.

It follows from (4.8) that a more explicit representation of the regime factor can be written as

$$
w_{t+1}=\alpha w_{t}+\underbrace{\rho_{z v} \epsilon_{z, t}+\rho_{g v} \epsilon_{g, t}+\rho_{R v} \epsilon_{R, t}}_{\text {endogenous drivers }}+\underbrace{\sqrt{1-\rho^{\prime} \rho} \eta_{t+1}}_{\text {exogenous driver }}, \quad \eta_{t+1} \sim \mathbb{N}(0,1)
$$

where the internal innovations $\left(\epsilon_{z, t}, \epsilon_{g, t}, \epsilon_{R, t}\right)$ and the external innovation $\eta_{t+1}$ are all orthogonal and have unit variance. Equation (4.10) asserts a complete separation between the three individual endogenous drivers $\left(\epsilon_{z}, \epsilon_{g}, \epsilon_{R}\right)$ and the exogenous driver $\eta$ of the regime factor. Also recall that $\left(\rho_{z v}^{2}, \rho_{g v}^{2}, \rho_{R v}^{2}, 1-\rho^{\prime} \rho\right)$ measure the percentage contributions of $\left(\epsilon_{z}, \epsilon_{g}, \epsilon_{R}, \eta\right)$ to the unconditional variance of $w$ and hence the extents to which these drivers trigger historical regime changes. In what follows, we quantify how much of the U.S. monetary policy shifts can be attributed, respectively, to each of technology growth, government spending, and monetary policy shocks.

4.2 Solution Method Equations (4.1)-(4.6) constitute a rational expectations system that can be cast into the generic form

$$
\mathbb{E}\left[f_{s_{t}}\left(x_{t+1}, x_{t}, x_{t-1}, \epsilon_{t}\right) \mid \mathcal{F}_{t}\right]=0
$$

where $s_{t}=1, \ldots, h$ is the regime at time $t, f_{s_{t}}$ a vector of nonlinear functions, $x_{t}$ a vector of model variables, and $\epsilon_{t}$ a vector of shock innovations. As mentioned earlier, private agents can observe current and all past realizations of endogenous variables, exogenous shocks, and regime states, but not the regime factors. Accordingly, they formulate rational expectations about future variables on the basis of the information set $\mathcal{F}_{t}=\sigma\left(\left\{x_{k}, \epsilon_{k}, s_{k}\right\}_{k=0}^{t}\right)$.

System (4.11) has to be solved before the model can be taken to data. To that end, a spate 
of theoretical and empirical efforts have managed to solve regime-switching rational expectations models using numerical techniques. One strand of the literature embraces the projection method to iteratively construct policy functions over a discretized state space [Davig (2004), Davig and Leeper (2006a,b), Bi and Traum (2012, 2014), Davig et al. (2010, 2011), Richter et al. (2014)]. Nevertheless, global approximations suffer from, among other problems, the curse of dimensionality that renders the practical implementation computationally costly even for small-scale models. The second strand begins with a linear or linearized model as if its parameters were constant and then annexes Markov switching to certain parameters [Svensson and Williams (2007), Farmer et al. (2011), Bianchi (2013), Cho (2016), Bianchi and Ilut (2017), Bianchi and Melosi (2017)]. While this approach is not as limited by the model size as the projection method, linearization without accounting for the switching parameters may be inconsistent with the optimizing behavior of agents who are aware of the switching process in the original nonlinear model. The third strand circumvents the above problems by embedding regime switching in perturbation solutions whose accuracy can be enhanced with higher-order terms [Maih (2015), Foerster et al. (2016), Barthélemy and Marx (2017), Bjørnland et al. (2018), Maih and Waggoner (2018)].

We obtain the model solution to the nonlinear rational expectations system (4.11) using the perturbation approach of Maih and Waggoner (2018), which is more general than the ones found in the earlier literature. As opposed to Foerster et al. (2016), for example, it requires no partition of the switching parameters, allows for the possibility of multiple steady states and, more importantly, can handle models with endogenous transition probabilities. ${ }^{15}$ To fix ideas, we seek a regime-specific policy function for $x_{t}$ that depends on the minimum set of state variables $\left(x_{t-1}, \epsilon_{t}\right)$

$$
x_{t}=g_{s t}\left(x_{t-1}, \epsilon_{t}\right)
$$

Substituting (4.12) into (4.11) and integrating out the future regime yield

$$
\mathbb{E}\left[\sum_{j=1}^{h} p_{i, j}\left(\epsilon_{t}\right) f_{i}\left(g_{j}\left(g_{i}\left(x_{t-1}, \epsilon_{t}\right), \epsilon_{t+1}\right), g_{i}\left(x_{t-1}, \epsilon_{t}\right), x_{t-1}, \epsilon_{t}\right) \mid \mathcal{F}_{t}\right]=0
$$

where the notational convention that $s_{t}=i$ and $s_{t+1}=j$ is followed. In general, there is no analytical solution to (4.13) even when $f_{i}$ is linear. Maih and Waggoner (2018) proposed to obtain a Taylor series approximation to (4.12) by introducing an auxiliary argument $\chi$ (i.e., the perturbation parameter)

$$
x_{t}=g_{i}\left(x_{t-1}, \epsilon_{t}, \chi\right)
$$

\footnotetext{
${ }^{15}$ Barthélemy and Marx (2017) also generalized standard perturbation methods to solve a class of nonlinear rational expectations models with endogenous regime switching.
} 
that solves a perturbed version of (4.13)

$$
\mathbb{E}\left[\sum_{j=1}^{h} q_{i, j}\left(\epsilon_{t}, \chi\right) f_{i}\left(g_{j}\left(h_{i}\left(x_{t-1}, \epsilon_{t}, \chi\right), \chi \epsilon_{t+1}, \chi\right), g_{i}\left(x_{t-1}, \epsilon_{t}, \chi\right), x_{t-1}, \epsilon_{t}\right) \mid \mathcal{F}_{t}\right]=0
$$

with perturbed transition probability $q_{i, j}\left(\epsilon_{t}, \chi\right)$ and perturbed policy function $h_{i}\left(x_{t-1}, \epsilon_{t}, \chi\right)$.

The principle of perturbation is to choose $q_{i, j}\left(\epsilon_{t}, \chi\right)$ and $h_{i}\left(x_{t-1}, \epsilon_{t}, \chi\right)$ such that $(4.15)$ becomes the original system (4.13) when $\chi=1$, but it reduces to a tractable and interpretable system when $\chi=0$. While (4.15) is already successful in eliminating the stochastic disturbances when $\chi=0$, choices of $q_{i, j}\left(\epsilon_{t}, \chi\right)$ and $h_{i}\left(x_{t-1}, \epsilon_{t}, \chi\right)$ still play a key role in interpreting the model's steady state $x_{i}=g_{i}\left(x_{i}, 0,0\right)$ around which the solution is expanded. In the context of exogenous Markov switching, Foerster et al. (2016) set $q_{i, j}\left(\epsilon_{t}, \chi\right)=p_{i, j}$ and $h_{i}\left(x_{t-1}, \epsilon_{t}, \chi\right)=g_{i}\left(x_{t-1}, \epsilon_{t}, \chi\right)$. Plugging their choice into (4.15) and evaluating it at the steady state give

$$
\sum_{j=1}^{h} p_{i, j} f_{i}\left(g_{j}\left(x_{i}, 0,0\right), x_{i}, x_{i}, 0\right)=0
$$

Since the only point at which we know how to evaluate $g_{j}$ is $\left(x_{j}, 0,0\right)$, this implies that $x_{i}=x_{j}$ for each $j$ and hence the steady state must be independent of any regime. ${ }^{16}$ By contrast, recognizing that switching parameters may imply distinct steady states, Maih and Waggoner's (2018) choice of

$$
q_{i, j}\left(\epsilon_{t}, \chi\right)= \begin{cases}\chi p_{i, j}\left(\epsilon_{t}\right), & i \neq j \\ \chi\left(p_{i, i}\left(\epsilon_{t}\right)-1\right)+1, & i=j\end{cases}
$$

and $h_{i}\left(x_{t-1}, \epsilon_{t}, \chi\right)=g_{i}\left(x_{t-1}, \epsilon_{t}, \chi\right)+(1-\chi)\left(x_{j}-x_{i}\right)$ implies

$$
f_{i}\left(x_{i}, x_{i}, x_{i}, 0\right)=0
$$

Consequently, $x_{i}$ can be readily interpreted as the deterministic steady state that would prevail in regime- $i$ when it is considered in isolation.

4.3 Econometric Method We estimate the model with Bayesian methods using a common set of quarterly observations, ranging from 1954:Q3 to 2007:Q4: per capita real output growth

\footnotetext{
${ }^{16}$ In this regard, the literature typically defines the steady state as the one associated with the ergodic mean values of Markov switching parameters. Such unique steady state, however, need not be an attractor-a resting point towards which the model tends to converge in the absence of further shocks. For example, Aruoba et al. (2017) presented a model that exhibits two distinct attractors, i.e., a targeted-inflation steady state and a deflationary steady state.
} 
(YGR), annualized inflation rate (INF), and effective federal funds rate (INT). ${ }^{17}$ The actual data are constructed as in Appendix B of Herbst and Schorfheide (2015) and available from the Federal Reserve Economic Data (FRED). The observable variables are linked to the model variables through the following measurement equations

$$
\left(\begin{array}{c}
\mathrm{YGR}_{t} \\
\mathrm{INF}_{t} \\
\mathrm{INT}_{t}
\end{array}\right)=\left(\begin{array}{c}
\gamma^{(Q)} \\
\pi^{(A)} \\
\pi^{(A)}+r^{(A)}+4 \gamma^{(Q)}
\end{array}\right)+100\left(\begin{array}{c}
\ln \left(z_{t} y_{t} / y_{t-1}\right) \\
4 \ln \left(\pi_{t} / \pi\right) \\
4 \ln \left(R_{t} / R\right)
\end{array}\right)
$$

where $\left(\gamma^{(A)}, \pi^{(A)}, r^{(A)}\right)$ are connected to the model's steady states via $\gamma=1+\gamma^{(A)} / 400, \beta=$ $1 /\left(1+r^{(A)} / 400\right)$, and $\pi=1+\pi^{(A)} / 400$. Let $\theta$ be a vector collecting all model parameters. In conjunction with the model solution, a first-order approximation to equations (4.16) and (4.14) around steady states maps directly into the general state space form (2.1)-(2.2), whose likelihood function $p\left(Y_{1: T} \mid \theta\right)$ can be evaluated with our endogenous-switching Kalman filter. ${ }^{18}$

In the Bayesian paradigm, the state space model is completed with a prior distribution $p(\theta)$ summarizing the researcher's initial views of the model parameters. This prior information is updated with the sample information via Bayes' theorem

$$
p\left(\theta \mid Y_{1: T}\right) \propto p\left(Y_{1: T} \mid \theta\right) p(\theta)
$$

where the posterior distribution $p\left(\theta \mid Y_{1: T}\right)$ characterizing the researcher's updated parameter beliefs is calculated up to the normalization constant. Since $p\left(\theta \mid Y_{1: T}\right)$ is typically intractable, one relies on Markov chain Monte Carlo methods to draw sample variates from it, which are then used to find a posterior summary of the distribution.

In practice, the solution algorithm of Maih and Waggoner (2018) and the estimation procedure have been coded up in RISE, a flexible object-oriented MATLAB toolbox developed by Junior Maih, that is well-suited for solving and estimating a general class of regime-switching DSGE models. ${ }^{19}$ See the Online Appendix for a user guide.

4.4 PrIORs Table 1 reports the priors of all structural parameters using $5 \%$ and $95 \%$ quantiles of the distributions. For the steady state parameters, the annualized growth rate $\gamma^{(A)}$ follows a normal distribution with quantiles 0.14 and 0.79 ; the annualized inflation rate $\pi^{(A)}$ follows a gamma distri-

\footnotetext{
${ }^{17}$ Our sample begins when the federal funds rate data first became available and ends before the federal funds rate nearly hit its effective lower bound.

${ }^{18}$ The standard stability concept for constant-parameter linear rational expectations models does not extend to the regime switching case. Instead, following the lead of Svensson and Williams (2007) and Farmer et al. (2011) among others, we adopt the concept of mean square stability to characterize first-order stable solutions.

${ }^{19}$ The toolbox is available, free of charge, at https://github.com/jmaih/RISE_toolbox.
} 
Table 1: Priors and Posteriors of DSGE Parameters

\begin{tabular}{|c|c|c|c|c|c|c|c|}
\hline \multirow[b]{2}{*}{ Parameter } & \multicolumn{3}{|c|}{ Prior } & \multicolumn{4}{|c|}{ Posterior } \\
\hline & Density & $5 \%$ & $95 \%$ & Mode & Median & $5 \%$ & $95 \%$ \\
\hline$\tau_{c}$ & $\mathbb{G}$ & 1.25 & 2.88 & 3.50 & 4.15 & 3.46 & 4.15 \\
\hline$\kappa$ & $\mathbb{G}$ & 0.06 & 0.38 & 0.45 & 0.63 & 0.43 & 0.63 \\
\hline$\psi_{y}$ & $\mathbb{G}$ & 0.17 & 0.96 & 0.18 & 0.07 & 0.07 & 0.18 \\
\hline$\rho_{z}$ & $\mathbb{B}$ & 0.33 & 0.66 & 0.95 & 0.94 & 0.94 & 0.95 \\
\hline$\rho_{g}$ & $\mathbb{B}$ & 0.33 & 0.66 & 0.95 & 0.94 & 0.94 & 0.95 \\
\hline$\rho_{R}$ & $\mathbb{B}$ & 0.33 & 0.66 & 0.77 & 0.75 & 0.75 & 0.77 \\
\hline$r^{(A)}$ & $\mathbb{G}$ & 0.34 & 0.67 & 0.46 & 0.54 & 0.46 & 0.54 \\
\hline$\pi^{(A)}$ & $\mathbb{G}$ & 1.23 & 7.60 & 2.57 & 2.30 & 2.30 & 2.57 \\
\hline$\gamma^{(A)}$ & $\mathbb{N}$ & 0.14 & 0.79 & 0.35 & 0.32 & 0.32 & 0.35 \\
\hline$\nu$ & $\mathbb{B}$ & 0.03 & 0.19 & 0.07 & 0.04 & 0.04 & 0.09 \\
\hline$c / y$ & $\mathbb{B}$ & 0.65 & 0.97 & 0.92 & 0.99 & 0.90 & 0.99 \\
\hline $100 \sigma_{z, 0}$ & $\mathbb{I} \mathbb{G}$ & 0.08 & 1.12 & 0.05 & 0.04 & 0.04 & 0.05 \\
\hline $100 \sigma_{z, 1}$ & $\mathbb{I} \mathbb{G}$ & 0.08 & 1.12 & 0.09 & 0.06 & 0.06 & 0.10 \\
\hline $100 \sigma_{g, 0}$ & $\mathbb{I} \mathbb{G}$ & 0.08 & 1.12 & 0.86 & 0.89 & 0.86 & 0.89 \\
\hline $100 \sigma_{g, 1}$ & $\mathbb{I} \mathbb{G}$ & 0.08 & 1.12 & 1.53 & 1.34 & 1.34 & 1.57 \\
\hline $100 \sigma_{R, 0}$ & $\mathbb{I} \mathbb{G}$ & 0.08 & 1.12 & 0.14 & 0.13 & 0.13 & 0.14 \\
\hline $100 \sigma_{R, 1}$ & $\mathbb{I} \mathbb{G}$ & 0.08 & 1.12 & 0.45 & 0.49 & 0.44 & 0.49 \\
\hline$p_{01}$ & $\mathbb{B}$ & 0.00 & 0.26 & 0.02 & 0.00 & 0.00 & 0.03 \\
\hline$p_{10}$ & $\mathbb{B}$ & 0.00 & 0.30 & 0.08 & 0.00 & 0.00 & 0.10 \\
\hline$\psi_{\pi, 0}$ & $\mathbb{G}$ & 0.84 & 1.17 & 0.93 & 0.92 & 0.91 & 0.93 \\
\hline$\psi_{\pi, 1}$ & $\mathbb{G}$ & 1.60 & 2.42 & 1.16 & 1.20 & 1.11 & 1.20 \\
\hline$\alpha$ & $\mathbb{B}$ & 0.80 & 0.96 & 0.93 & 0.86 & 0.86 & 0.93 \\
\hline$\tau$ & $\mathbb{N}$ & -1.82 & -0.17 & -0.13 & -0.05 & -0.12 & -0.05 \\
\hline$\rho_{z v}$ & $\mathbb{U}$ & -0.90 & 0.90 & -0.16 & 0.01 & -0.21 & 0.01 \\
\hline$\rho_{g v}$ & $\mathbb{U}$ & -0.90 & 0.90 & 0.70 & 0.88 & 0.67 & 0.88 \\
\hline$\rho_{R v}$ & $\mathbb{U}$ & -0.90 & 0.90 & -0.63 & -0.46 & -0.63 & -0.46 \\
\hline
\end{tabular}

Notes: The following abbreviations are used: Gamma distribution $(\mathbb{G})$, Normal distribution $(\mathbb{N})$, Beta distribution $(\mathbb{B})$, and Inverse-Gamma type-I distribution $(\mathbb{I} G)$.

bution with quantiles 1.23 and 7.60; and the annualized real rate $r^{(A)}$ follows a gamma distribution with quantiles 0.34 and 0.67 . The priors on the structural shock processes are harmonized: the autoregressive coefficients $\left(\rho_{z}, \rho_{g}, \rho_{R}\right)$ are beta distributed with quantiles 0.33 and 0.66 , and the standard deviation parameters $\left(\sigma_{z}\left(s_{t}^{\mathrm{Vol}}\right), \sigma_{g}\left(s_{t}^{\mathrm{Vol}}\right), \sigma_{R}\left(s_{t}^{\mathrm{Vol}}\right)\right)$, all scaled by 100 , follow inverse-gamma 
type-I distribution with quantiles 0.08 and 1.12. Furthermore, the priors on the private sector parameters $\left(\tau_{c}, \kappa, \nu, c / y\right)$ and the policy response to output $\psi_{y}$ are largely adopted from An and Schorfheide (2007), whereas those on the policy responses to inflation $\left(\psi_{\pi, 0}, \psi_{\pi, 1}\right)$ closely follow the specification in Davig and Doh (2014), which a priori rule out the possibility of 'label switching' by imposing $\psi_{\pi, 0}<\psi_{\pi, 1}$ and hence achieve regime identification. Finally, turning to the parameters for the autoregressive regime factor, the prior on $\alpha$ centers at a rather persistent value that, together with the prior mean of $\tau$, implies symmetric transition probabilities $(0.85,0.85)$ under exogenous switching. On the other hand, the uniform priors on $\left(\rho_{z v}, \rho_{g v}, \rho_{R v}\right)$ centering at zero reflect an agnostic view about the sign and degree of endogeneity in regime switching.

4.5 Posterior Estimates We adopt the stochastic search optimization routines of the RISE toolbox to estimate the posterior mode. With a mode or starting point in hand, we sample a total of one million draws from the posterior distribution using the Metropolis-Hastings algorithm and save every fifth draw. The resulting 200,000 draws form the basis for performing our posterior inference. We highlight several aspects of the posterior estimates reported in Table 1 as follows.

First, the $90 \%$ credible intervals show that the posterior distributions for most of the parameters are different from those implied by the prior. Hence, the data are informative about most of the estimated parameters. Second, turning to the policy switching parameters, the overall monetary response to inflation appears to be much less aggressive than assumed a priori- the $90 \%$ posterior interval of the more active response $\psi_{\pi, 1}$ is shifted significantly downward. Third, moving to the parameters $(\alpha, \tau)$ unique to our threshold switching, a prior-posterior comparison reveals that the data support a persistent process for the regime factor, and only slightly favor the more active regime by making it relatively easier for the regime factor to remain above the threshold. Finally, the posterior mode of $\rho$ attributes a significant portion of regime developments to past government spending shocks (about 49\%) and monetary policy shocks (about 40\%), and to a lesser extent, technology growth shocks (about 3\%).

A prior-posterior comparison of the correlation parameters $\left(\rho_{z v}, \rho_{g v}, \rho_{R v}\right)$ further substantiates the relevance of accounting for the endogenous feedback from historical macroeconomic shocks to the prevailing policy regime. Despite the diffuse priors, the data turn out to be informative to land the posteriors onto narrower areas of the parameter space that deliver tightly estimated degrees of endogeneity in regime switching. Most noticeable is the endogenous feedback from government spending shock, whose posterior mass falls entirely on the positive territory. As a result, favorable demand disturbances will increase the likelihood of staying in or shifting to the more active regime, consistent with a countercyclical monetary policy that is 'leaning against the wind'. Also evident is the posterior distribution of the endogenous feedback from monetary policy shock that concentrates entirely on the negative territory. Thus, contractionary monetary shocks will decrease the likelihood of staying in or shifting to the more active regime in the future, which is somewhat consistent with the low estimate of the more active response $\psi_{\pi, 1}$ to inflation. Technology growth 

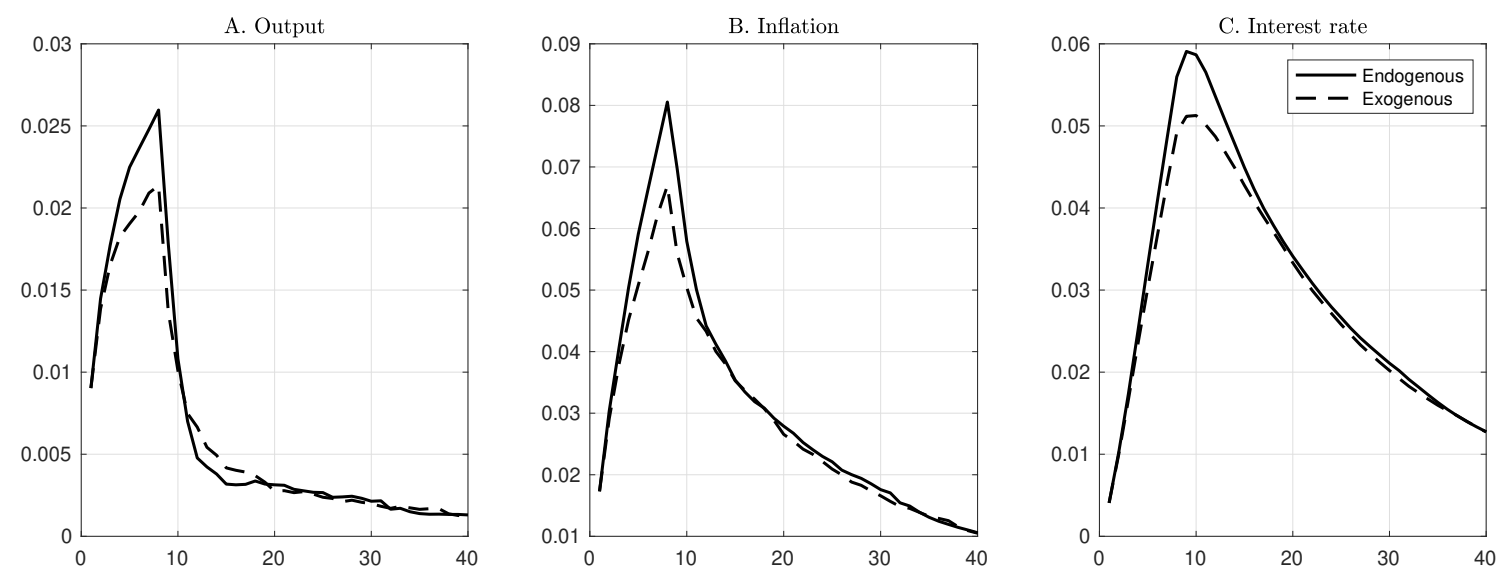

Figure 5: Dynamic responses to technology growth shocks at the posterior mode under endogenous switching. Notes: Panels A-C plot the projected paths of key endogenous variables conditional on a twoyear sequence of technology growth shocks $\{4,4,4,4,4,4,4,4,0, \ldots, 0\}$. The exogenous-switching response is obtained by setting $\rho_{z v}=\rho_{g v}=\rho_{R v}=0$ while keeping other parameters unchanged. All variables are in level deviations from steady state.

shock, on the other hand, plays a less evident role in driving the regime changes. In particular, favorable technological advancements tend to make the less active regime more likely, suggesting an accommodative monetary policy to promote long-term economic growth.

Just as the analytical example of Section 3 demonstrates, endogenizing regime changes here can generate important expectations-formation effects beyond what the exogenous switching can do. For example, consider a two-year sequence of positive technology shocks $\{4,4,4,4,4,4,4,4,0, \ldots, 0\}$. Figure 5 compares the conditional forecasts of key model variables under endogenous and exogenous switching. ${ }^{20}$ As in the fixed regime, higher productivity raises both aggregate activity and (through the Phillips curve) inflation, which in turn leads to a rise in interest rate according to the monetary policy rule. It also triggers a negative feedback effect $\left(\rho_{z v}=-0.16\right)$ that induces agents to form a stronger belief in the less active regime under which output and inflation rise by even more. This shift in agents' expectations spells out the discrepancies in the forecasts under exogenous and endogenous switching (i.e., with and without the restriction $\rho_{z v}=0$ ).

Unlike the Markov switching filter of Kim (1994), our filter also produces an important byproduct, i.e., an estimated time series of the regime factor $w_{t \mid t}$. Loosely speaking, this series conveniently packs, through a reduced-form mechanism, various structural and extraneous sources

\footnotetext{
${ }^{20}$ Because the model is inherently nonlinear and does not admit an analytical solution, sequential Monte Carlo methods are required to numerically integrate out the regime index in each period. To compute the conditional forecasts, we simulate $N=1000$ trajectories of regime states $\left\{s_{T+1}^{(i)}, \ldots, s_{T+K}^{(i)}\right\}_{i=1}^{N}$, apply the regime-specific policy function to obtain the corresponding model variables $\left\{x_{T+1}^{(i)}, \ldots, x_{T+K}^{(i)}\right\}_{i=1}^{N}$ conditional on a pre-specified sequence of technology shocks $\left\{\epsilon_{u, T+1}, \ldots, \epsilon_{u, T+K}\right\}$ but zeroing out all other shocks, and take the sample average of $\left\{x_{t}^{(i)}\right\}_{i=1}^{N}$ for $t=T+1, \ldots, T+K$. In the special case of one-period shock $\{1,0, \ldots, 0\}$, these forecasts are equivalent to the generalized impulse response functions of Koop et al. (1996).
} 
A. Estimated Regime Factor

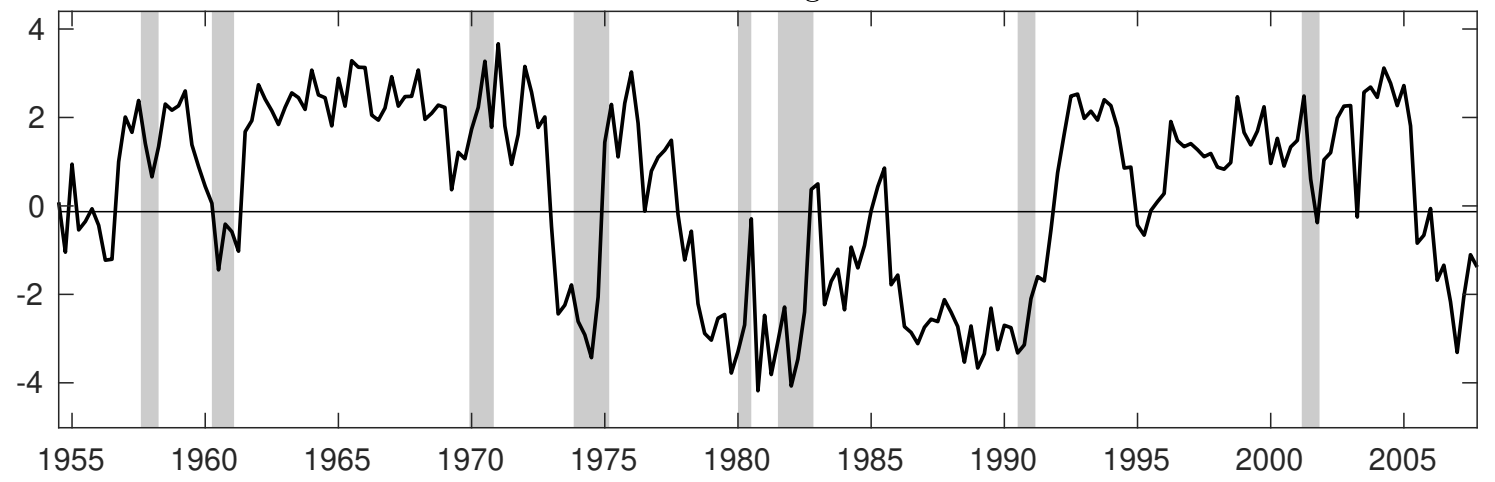

B. Estimated High-Vol Probability

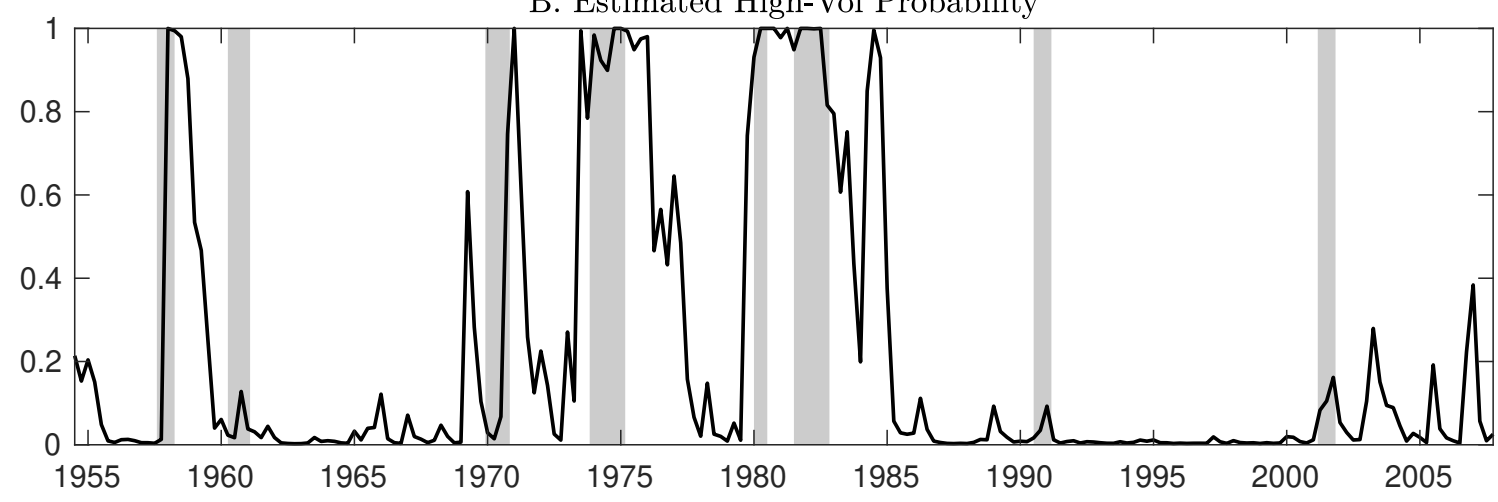

Figure 6: Filtered regime factor and high-volatility probability at the posterior mode under endogenous switching. Notes: The horizontal line in Panel A delineates the threshold level. Shaded bars indicate recessions as designated by the National Bureau of Economic Research.

of regime changes into one single index that is likely to proxy for some endogenous economic variables relevant to the regime generating process. We refer the reader to Chang et al. (2019) who corroborate this interpretation by linking the estimated policy regime factor to a large pool of macro and financial variables and selecting the variables that have significant explanatory power.

As can be seen from Panel A of Figure 6, the estimated regime factor exhibits prolonged swings that identify the U.S. monetary policy as sluggishly fluctuating between the more active and less active regimes, the timing and nature of which are roughly consistent with the previous empirical findings. Such a pattern also aligns somewhat with the narrative record of policymakers' beliefs documented in Romer and Romer (2004): the more active stance of the late 1950s and most of the 1960s under chairman William McChesney Martin Jr. and of the mid-1980s and beyond under Paul Volcker and Alan Greenspan stemmed from the conviction that inflation has high costs and few benefits; the less active stance of most of the 1970s under Arthur Burns and G. William Miller stemmed from an overestimate of the natural rate of unemployment as well as an underestimate of the sensitivity of inflation to economic slack. On the other hand, the estimated probability of being in the high volatility regime (Panel B) identifies periods of heightened shock volatility as mostly 
coinciding with the NBER recessions.

\section{Concluding Remarks}

This paper aims at broadening the scope for understanding the complex interaction between recurrent regime changes and measured economic behavior. To that end, we introduce a threshold-type endogenous regime switching into an otherwise standard state space model, which is general enough to encompass many well-known dynamic linear models, including classical regression models and the popular DSGE models as special cases. In our approach, regime changes are, through an autoregressive latent factor, jointly driven by the internal innovations that represent the fundamental shocks inside the model and an external innovation that captures all other shocks left outside of the model. This allows the behavior of underlying economic fundamentals to bear more directly on the regime generating process. When regime switches are purely driven by the external innovation, our model reduces to one with exogenous Markov switching.

We develop an endogenous switching version of the Kalman filter to estimate the overall nonlinear state space model. This filter features augmenting the transition system with the internal innovations that, in conjunction with a collapsing procedure to truncate the regime history, makes the computation feasible and efficient. For pedagogical purposes, we employ the filter to estimate the state space representation of a prototypical new Keynesian DSGE model with threshold switching in monetary policy rule and Markov switching in shock volatility, and illustrate how the underlying structural shocks impact agents' expectations formation and monetary policy regimes through our novel endogenous feedback channel. We find compelling statistical support for the endogenous feedback from historical shocks to the prevailing policy regime.

We remind the reader that quantifying such feedback mechanism depends on nearly every aspect of private and policy behavior. A comprehensive investigation calls for a richly structured mediumscale DSGE model with fiscal details in both the model specification and the observable data. Another natural extension of our framework is to permit multiple regimes and latent factors along with the development of its filtering algorithm. We defer these extensions to a sequel to this paper.

\section{ACKNOWLEDGEMENTS}

The authors would like to thank seminar and conference participants at Australian National University, Central University of Finance and Economics, Keio University, Korea Development Institute, MIT-Harvard, North Carolina State University, Renmin University of China, the Reserve Bank of Australia, Toulouse School of Economics, the University of California-Santa Barbara, the University of Chicago Booth School of Business, the University of Sydney, 2017 NBER Workshop on DSGE Models at Philadelphia Fed, 2018 Workshop on "Nonlinear Models in Macroeconomics and Finance for an Unstable World" at Norges Bank, 2018 International Association for Applied Econometrics 
Annual Conference at Montréal, 2018 Taiwan Econometric Society Annual Meeting, the 27th Annual Symposium of the Society for Nonlinear Dynamics and Econometrics at Dallas, and the 29th Annual Meeting of the Midwest Econometrics Group at Columbus, as well as Francesco Bianchi, Yongok Choi, Olivier Coibion, Luca Guerrieri, Edward Herbst, Eric Leeper, Chaojun Li, Joon Park, Shi Qiu, Daniel Waggoner, and Tao Zha for very useful and stimulating comments. Fei Tan acknowledges financial support from the Chaifetz School of Business summer research grant.

\section{ONLINE APPENDIX}

Appendix A: Transition Probability The prerequisite to computing transition probabilities is to evaluate integrals of the form

$$
\begin{aligned}
F(a, b, c, d, m, V) & =\int_{-\infty}^{\infty} \int_{-\infty}^{a} \Phi(b+c x+d y) p_{\mathbb{N}}(x \mid 0,1) p_{\mathbb{N}}(y \mid m, V) d x d y \\
& =\mathbb{P}\left(Z_{1} \leqslant a, Z_{2} \leqslant b+c Z_{1}+d Z_{3}\right)
\end{aligned}
$$

for some $a, b, c, d \in \mathbb{R}$, where $Z_{1}, Z_{2} \sim \mathbb{N}(0,1), Z_{3} \sim \mathbb{N}(m, V)$, and $\left(Z_{1}, Z_{2}, Z_{3}\right)$ are independent of each other. Define $W_{1}=Z_{1} \sim \mathbb{N}(0,1)$ and $W_{2}=Z_{2}-d Z_{3} \sim \mathbb{N}\left(-d m, 1+d^{2} V\right)$. Then $\left(W_{1}, W_{2}\right)$ are independent and (A.1) can be rewritten as

$$
\begin{aligned}
F(a, b, c, d, m, V) & =\mathbb{P}\left(W_{1} \leqslant a, W_{2}-c W_{1} \leqslant b\right) \\
& =\int_{-\infty}^{b} \int_{-\infty}^{a} p_{\mathbb{N}}(x, y \mid \mu, \Sigma) d x d y
\end{aligned}
$$

where

$$
\mu=\left(\begin{array}{c}
0 \\
-d m
\end{array}\right), \quad \Sigma=\left(\begin{array}{cc}
1 & -c \\
-c & 1+c^{2}+d^{2} V
\end{array}\right)
$$

Therefore, the expected transition probabilities in Algorithm 1 of Section 2.2 can be calculated as

$$
\begin{aligned}
& \int_{-\infty}^{\infty} \mathbb{P}\left(s_{t}=0 \mid s_{t-1}=0, \lambda_{t-1}, \mathcal{F}_{t-1}\right) p\left(\lambda_{t-1} \mid s_{t-1}=0, \mathcal{F}_{t-1}\right) d \lambda_{t-1} \\
= & \frac{1}{\Phi\left(\tau \sqrt{1-\alpha^{2}}\right)} F\left(\tau \sqrt{1-\alpha^{2}}, \frac{\tau}{\sqrt{1-\rho^{\prime} \rho}},-\frac{\alpha}{\sqrt{1-\rho^{\prime} \rho} \sqrt{1-\alpha^{2}}},-\frac{1}{\sqrt{1-\rho^{\prime} \rho}}, \rho^{\prime} \varsigma_{d, t-1 \mid t-1}^{0}, \rho^{\prime} P_{d, t-1 \mid t-1}^{0} \rho\right)
\end{aligned}
$$


and

$$
\begin{aligned}
& \int_{-\infty}^{\infty} \mathbb{P}\left(s_{t}=0 \mid s_{t-1}=1, \lambda_{t-1}, \mathcal{F}_{t-1}\right) p\left(\lambda_{t-1} \mid s_{t-1}=1, \mathcal{F}_{t-1}\right) d \lambda_{t-1} \\
= & \frac{1}{1-\Phi\left(\tau \sqrt{1-\alpha^{2}}\right)} F\left(-\tau \sqrt{1-\alpha^{2}}, \frac{\tau}{\sqrt{1-\rho^{\prime} \rho}}, \frac{\alpha}{\sqrt{1-\rho^{\prime} \rho} \sqrt{1-\alpha^{2}}},-\frac{1}{\sqrt{1-\rho^{\prime} \rho}}, \rho^{\prime} \varsigma_{d, t-1 \mid t-1}^{1}, \rho^{\prime} P_{d, t-1 \mid t-1}^{1} \rho\right)
\end{aligned}
$$

Moreover, the expected transition probabilities in Section 3.3 can be calculated as

$$
\mathbb{E} p_{00}\left(\epsilon_{i, t}\right)=\frac{1}{\Phi\left(\tau \sqrt{1-\alpha^{2}}\right)} F\left(\tau \sqrt{1-\alpha^{2}}, \frac{\tau}{\sqrt{1-\rho^{2}}},-\frac{\alpha}{\sqrt{1-\rho^{2}} \sqrt{1-\alpha^{2}}},-\frac{\rho}{\sqrt{1-\rho^{2}}}, 0,1\right)
$$

and

$$
\mathbb{E} p_{10}\left(\epsilon_{i, t}\right)=\frac{1}{1-\Phi\left(\tau \sqrt{1-\alpha^{2}}\right)} F\left(-\tau \sqrt{1-\alpha^{2}}, \frac{\tau}{\sqrt{1-\rho^{2}}}, \frac{\alpha}{\sqrt{1-\rho^{2}} \sqrt{1-\alpha^{2}}},-\frac{\rho}{\sqrt{1-\rho^{2}}}, 0,1\right)
$$

Lastly, the transition probabilities in Section 3.2 can be calculated as

$$
p_{00}\left(\epsilon_{t}\right)=\frac{1}{\Phi\left(\tau \sqrt{1-\alpha^{2}}\right)} F\left(\tau \sqrt{1-\alpha^{2}}, \frac{\tau-\rho^{\prime} \epsilon_{t}}{\sqrt{1-\rho^{\prime} \rho}},-\frac{\alpha}{\sqrt{1-\rho^{\prime} \rho} \sqrt{1-\alpha^{2}}}, 0, \cdot, \cdot\right)
$$

and

$$
p_{10}\left(\epsilon_{t}\right)=\frac{1}{1-\Phi\left(\tau \sqrt{1-\alpha^{2}}\right)} F\left(-\tau \sqrt{1-\alpha^{2}}, \frac{\tau-\rho^{\prime} \epsilon_{t}}{\sqrt{1-\rho^{\prime} \rho}}, \frac{\alpha}{\sqrt{1-\rho^{\prime} \rho} \sqrt{1-\alpha^{2}}}, 0, \cdot, \cdot\right)
$$

ApPendix B: User GuIDE This is a brief tutorial that provides information for implementing the perturbation approach of Maih and Waggoner (2018) to solve the empirical model in Section 4.1 .

B.1 RISE Model File It is the file as07_cmt with extension .rs that contains declarations of endogenous variables, exogenous variables and parameters. Those atoms are respectively declared using keywords @endogenous, @exogenous and @parameters. All declarations are separated with a comma and/or with one or more white spaces. All exogenous variables (shocks) are assumed to be normally distributed with mean zero and standard deviation 1 . This assumption is not restrictive as those variables can always be scaled by a parameter.

Whenever a parameter is switching, we have to attach it to a Markov process controlling its behavior and declare the number of states for the parameter, e.g., Oparameters (vol,3) siga sigb means that Markov process vol has three states and controls the behavior of siga and sigb. Those parameters switch in locksteps. A parameter cannot be controlled by two or more Markov processes.

There could be many Markov processes in each model. Each Markov process gives rise to tran- 
sition probabilities that are either constant (exogenous switching) or time-varying (endogenous switching). Transition probabilities follow a specific syntax with exactly three underscores, e.g., vol_tp_1_3 is the transition probability in Markov process vol, for going from state 1 in the current period to state 3 in the next period.

Constant transition probabilities are declared as parameters while time-varying transition probabilities are endogenous variables with equations defined in the model block (see below).

For a Markov process in $h$ states $(h>1)$, only $h \times(h-1)$ transition probabilities need to be declared (exogenous switching) or given equations for (endogenous switching). The missing $h$ transition probabilities are the ones on the diagonal of the transition matrix, i.e., the probabilities of staying in each state. RISE does not allow those to be defined.

The keyword for declaring the model block is @model. The model block contains the structural equations for the system as well as, possibly, equations for time-varying transition probabilities in case there are some. It may also contain local definitions. These are preceeded by a \# sign. The equations for time-varying transition probabilities are preceeded by a! sign. All equations end with a semicolon.

If one wants to conduct a filtering or a filtering-based exercise, observable variables have to be declared. The keyword for declaring observable variables is @observables.

B.2 Differentiation Because RISE uses perturbation as the main solution strategy, it needs to be able to take derivatives of each function. The symbolic and automatic differentiators in RISE are prepared to take derivatives of most common functions : abs, acos, acosh, \&, asin, asinh, atan, atanh, cos, cosh, cot, eq, erf, exp, ge, gt, le, log, log10, lt, max, min, minus, plus, mpower, mrdivide, mtimes, ne, normcdf, normpdf, or, power, rdivide, sign, sin, sinh, sqrt, tan, tanh, times, uminus, uplus, and combinations of those.

However, in some applications the user may have a function that does not belong in the list above. In our application, this is the case for a function we named yoosoon.m, which computes the integrals entering the calculation of our time-varying transition probabilities. Whenever we face a situation where we are in the presence of a function that RISE does not know how to differentiate, the user has to provide the derivatives of the function up to the order of approximation desired.

Suppose the alien function takes $k$ arguments. Then it should be written in such a way that it accepts another two arguments, i.e., $k+2$ arguments in total. Whenever RISE wants to evaluate the function, it will call it with $k$ arguments. But whenever it wants to compute a derivative, the function will be called with $k+2$ arguments. Argument number $k+1$ is always 'diff', while argument number $k+2$ is the order of differentiation. Therefore, $\mathrm{y}=\mathrm{yoosoon}(\mathrm{z}, \mathrm{a}, \mathrm{L}, \mathrm{H})$ will evaluate the function whereas dy=yoosoon ( $z, a, L, H$, 'diff', 1 ) will compute the first-order derivative (with respect to the first argument), dy2=yoosoon ( $z, a, L, H$, 'diff', 2) will compute the second-order derivate, and dyn=yoosoon $(z, a, L, H$, 'diff',$n)$ will compute the nth-order derivative. 
B.3 Constructing a RISE Model Object When the RISE toolbox is loaded, a model object is constructed by issuing the statement $\mathrm{m}=\mathrm{rise}$ (modelFilename), where in the current example, modelFilename='as07_cmt'.

In the presence of alien functions, those functions need to be declared to RISE at the construction of the model object. The syntax $\mathrm{m}=\mathrm{rise}$ (modelFilename) now becomes $\mathrm{m}=\mathrm{rise}$ (modelFilename, 'alien_list', listOfAliens), where in our application listOfAliens='yoosoon', but could in general be a cell array of strings.

B.4 Solving a (Parameterized) Model in the Presence of Regime Switching Without regime switching, we solve a model by issuing the command $\mathrm{m}=\mathrm{solve}(\mathrm{m})$, provided that the model is parameterized and that the steady state is either provided or easy to compute numerically. The solving of the model in this way involves a standard perturbation, which turns off the effect of the uncertainty stemming from future shocks. ${ }^{21}$

In the presence of regime switching, an advanced perturbation strategy is called for. In addition to turning of the uncertainty from future shocks, this perturbation also has to turn off the uncertainty coming from a different type of shocks: markov processes. Two main perturbation strategies are available. The one we use in this paper is the one by Maih and Waggoner (2018) as described in the main text. We instruct RISE to choose that perturbation strategy by issuing the command m=solve (m, 'solve_perturbation_type' , 'mw').

By default, RISE solves a regime switching model using functional iteration. In the presence of the Maih-Waggoner perturbation, there are computational gains to be had. RISE implements two solvers to accompany the Maih-Waggoner perturbation. One of them with name 'dsge_schur' uses the generalized Schur decomposition and the other one with name 'dsge_udc' uses the method of undetermined coefficients. To add a particular solver, use the command m=solve(m, 'solver', solverName), where solverName could be 'mn' (Newton solver), 'mfi' (functional iteration), 'fwz' (Farmer et al. (2011)), 'dsge_udc', 'dsge_schur', etc.

B.5 Files Used in the PAPer The core files used in this paper are

- as07_cmt.rs: the model file.

- ass_cmt_ssfile.m: the steady state file.

- yoosoon.m: a helper routine for computing the transition probabilities.

- as07_data.txt: the data file, with variables later transformed into time series.

- as07_cmt_para.m: a function that sets the parameters as well as the priors for estimation.

\footnotetext{
${ }^{21}$ In this application, the program that solves the steady state is ass_cmt_ssfile.m.
} 
All other files are auxiliary functions written to facilitate multiple runs, data preparation, model comparisons, runs in parallel, plotting, etc. Those are

- set_solvers_and_filters.m

- set_parameters_and_priors.m

- set_directories.m

- sample_from_the_posterior.m

- rise_model.m

- driver_posterior_simulation.m

- driver_mcmc_mdd.m

- driver_convergence.m

- driver_as07_cmt_20200611.m

\section{REFERENCES}

An, S., And F. SchorfheIde (2007): "Bayesian Analysis of DSGE Models," Econometric Reviews, 26(2), 113-172.

Aruoba, S. B., P. Cuba-Borda, and F. Schorfheide (2017): "Macroeconomic Dynamics Near the ZLB: A Tale of Two Countries," The Review of Economic Studies, 85(1), 87-118.

BARThÉLEMy, J., AND M. MARX (2017): "Solving endogenous regime switching models," Journal of Economic Dynamics and Control, 77, 1-25.

Bazzi, M., F. Blasques, S. J. Koopman, and A. Lucas (2014): "Time-Varying Transition Probabilities for Markov Regime Switching Models," Journal of Time Series Analysis, 38(3), 458-478.

Benigno, G., A. T. Foerster, C. Otrok, and A. Rebucci (2020): "Estimating Macroeconomic Models of Financial Crises: An Endogenous Regime-Switching Approach," Staff Reports 944, Federal Reserve Bank of New York.

Best, G., And J. Hur (2019): "Bad Luck, Bad Policy, and Learning? A Markov-Switching Approach to Understanding Postwar U.S. Macroeconomic Dynamics," European Economic Review, forthcoming. 
Bi, H., and N. Traum (2012): "Estimating Sovereign Default Risk," The American Economic Review: Papers \& Proceedings, 102(3), 161-166.

- (2014): "Estimating Fiscal Limits: The Case of Greece," Journal of Applied Econometrics, $29(7), 1053-1072$.

Bianchi, F. (2013): "Regime Switches, Agents' Beliefs, and Post-World War II U.S. Macroeconomic Dynamics," The Review of Economic Studies, 80(2), 463-490.

Bianchi, F., And C. Ilut (2017): "Monetary/Fiscal Policy Mix and Agent's Beliefs," Review of Economic Dynamics, 26, 113-139.

Bianchi, F., And L. Melosi (2017): "Escaping the Great Recession," American Economic Review, 107(4), 1030-58.

Binning, A., And J. Main (2017): "Modelling Occasionally Binding Constraints Using RegimeSwitching," Working Paper 2017/23, Norges Bank.

Buørnland, H. C., V. H. Larsen, and J. Maih (2018): "Oil and Macroeconomic (In)stability," American Economic Journal: Macroeconomics, 10(4), 128-51.

Chang, Y., Y. Choi, and J. Y. PARK (2017): "A New Approach to Model Regime Switching," Journal of Econometrics, 196(1), 127 - 143.

Chang, Y., B. Kwak, and S. Qiu (2019): "U.S. Monetary and Fiscal Policy Regime Changes and Their Interactions," Manuscript.

Chang, Y., F. Tan, and X. Wei (2018a): "State Space Models with Endogenous Regime Switching," Manuscript.

(2018b): "A Structural Investigation of Monetary Policy Shifts," Manuscript.

Chen, R., And J. S. Liu (2000): "Mixture Kalman Filters," J. R. Statist. Soc. B, 62, 493-508.

Chib, S. (1996): "Calculating Posterior Distributions and Modal Estimates in Markov Mixture Models," Journal of Econometrics, 75, 79-97.

Chib, S., And M. J. Dueker (2004): "Non-Markovian regime switching with endogenous states and time-varying state strengths," Working Papers 2004-030, Federal Reserve Bank of St. Louis.

Cho, S. (2016): "Sufficient Conditions for Determinacy in a Class of Markov-Switching Rational Expectations Models," Review of Economic Dynamics, 21, 182 - 200. 
Clarida, R., J. Galí, and M. Gertler (2000): "Monetary Policy Rules and Macroeconomic Stability: Evidence and Some Theory," Quarterly Journal of Economics, 115(1), 147-180.

Davig, T. (2004): "Regime-Switching Debt and Taxation," Journal of Monetary Economics, 51(4), $837-859$.

Davig, T., And T. Doh (2014): "Monetary Policy Regime Shifts and Inflation Persistence," The Review of Economics and Statistics, 96(5), 862-875.

Davig, T., And E. M. Leeper (2006a): "Endogenous Monetary Policy Regime Change," in NBER International Seminar on Macroeconomics 2006, ed. by L. Reichlin, and K. D. West, pp. 345-377. MIT Press, Cambridge, MA.

(2006b): "Fluctuating Macro Policies and the Fiscal Theory," in NBER Macroeconomics Annual 2006, ed. by D. Acemoglu, K. Rogoff, and M. Woodford, vol. 21, pp. 247-298. MIT Press, Cambridge.

Davig, T., E. M. Leeper, and T. B. Walker (2010): “Unfunded Liabilities' and Uncertain Fiscal Financing," Journal of Monetary Economics, 57(5), 600-619.

(2011): "Inflation and the Fiscal Limit," European Economic Review, 55(1), 31-47.

Diebold, F. X., J.-H. Lee, and G. Weinbach (1994): "Regime switching with time-varying transition probabilities," in Nonstationary Time Series Analysis and Cointegration, ed. by C. Hargreaves, pp. 283-302. Oxford University Press.

FArmer, R. E., D. F. Waggoner, And T. Zha (2011): "Minimal state variable solutions to Markov-switching rational expectations models," Journal of Economic Dynamics and Control, 35(12), 2150 - 2166, Frontiers in Structural Macroeconomic Modeling.

Foerster, A., J. F. Rubio-Ramírez, D. F. Waggoner, and T. Zha (2016): "Perturbation methods for Markov-switching dynamic stochastic general equilibrium models," Quantitative Economics, 7(2), 637-669.

Guerrieri, L., And M. IAcoviello (2015): "OccBin: A toolkit for solving dynamic models with occasionally binding constraints easily," Journal of Monetary Economics, 70, 22 - 38.

(2017): "Collateral constraints and macroeconomic asymmetries," Journal of Monetary Economics, 90, $28-49$.

Hamilton, J. D. (1988): "Rational-expectations econometric analysis of changes in regime: An investigation of the term structure of interest rates," Journal of Economic Dynamics and Control, $12(2), 385-423$. 
(1989): "A New Approach to the Economic Analysis of Nonstationary Time Series and the Business Cycle," Econometrica, 57(2), 357-384.

Herbst, E. P., And F. Schorfheide (2015): Bayesian Estimation of DSGE Models. Princeton University Press.

Kalliovirta, L., M. Meitz, and P. Saikkonen (2015): "A Gaussian Mixture Autoregressive Model for Univariate Time Series," Journal of Time Series Analysis, 36(2), 247-266.

KANG, K. H. (2014): "Estimation of state-space models with endogenous Markov regime-switching parameters," The Econometrics Journal, 17(1), 56-82.

Kim, C.-J. (1994): "Dynamic Linear Models with Markov-Switching," Journal of Econometrics, $60(1), 1-22$.

(2004): "Markov-Switching Models with Endogenous Explanatory Variables," Journal of Econometrics, 122(1), 127-136.

(2009): "Markov-switching models with endogenous explanatory variables II: A two-step MLE procedure," Journal of Econometrics, 148(1), 46-55.

Kim, C.-J., And J. Kim (2018): "Non-Markovian Regime-Switching Models," Manuscript.

Kim, C.-J., And C. R. Nelson (1999): State-Space Models with Regime Switching. MIT Press, Cambridge, MA.

Kim, C.-J., J. Piger, And R. Startz (2008): "Estimation of Markov regime-switching regression models with endogenous switching," Journal of Econometrics, 143(2), 263-273.

Koop, G., M. Pesaran, and S. M. Potter (1996): "Impulse Response Analysis in Nonlinear Multivariate Models," Journal of Econometrics, 74(1), 119 - 147.

Leeper, E. M. (1991): "Equilibria Under 'Active' and 'Passive' Monetary and Fiscal Policies," Journal of Monetary Economics, 27(1), 129-147.

Leeper, E. M., And T. Zha (2003): "Modest Policy Interventions," Journal of Monetary Economics, 50(8), 1673-1700.

Liu, Z., D. F. Waggoner, And T. Zha (2011): "Sources of macroeconomic fluctuations: A regime-switching DSGE approach," Quantitative Economics, 2(2), 251-301.

MAin, J. (2015): "Efficient Perturbation Methods for Solving Regime-Switching DSGE Models," Working Paper 2015/01, Norges Bank. 
Maih, J., And D. Waggoner (2018): "Perturbation Methods for DSGE Models with TimeVarying Coefficients and Transition Matrices," Mimeograph, Norges Bank.

Richter, A., N. Throckmorton, and T. Walker (2014): “Accuracy, Speed and Robustness of Policy Function Iteration," Computational Economics, 44, 445?476.

Romer, C. D., and D. H. Romer (2004): "Choosing the Federal Reserve Chair: Lessons from History," Journal of Economic Perspectives, 18(1), 129-62.

Schorfheide, F. (2005): "Learning and Monetary Policy Shifts," Review of Economic Dynamics, $8(2), 392-419$.

Svensson, L. E. O., and N. Williams (2007): "Monetary Policy with Model Uncertainty: Distribution Forecast Targeting," CEPR Discussion Paper No. DP6331.

Tanner, M. A., And W. H. Wong (1987): "The Calculation of Posterior Distributions by Data Augmentation," Journal of the American Statistical Association, 82(398), 528-540.

TAYlor, J. B. (1993): "Discretion versus Policy Rules in Practice," Carneige-Rochester Conference Series on Public Policy, 39, 195-214.

Wei, G. C. G., And M. A. Tanner (1990): "A Monte Carlo Implementation of the EM Algorithm and the Poor Man's Data Augmentation Algorithms," Journal of the American Statistical Association, 85(411), 699-704. 